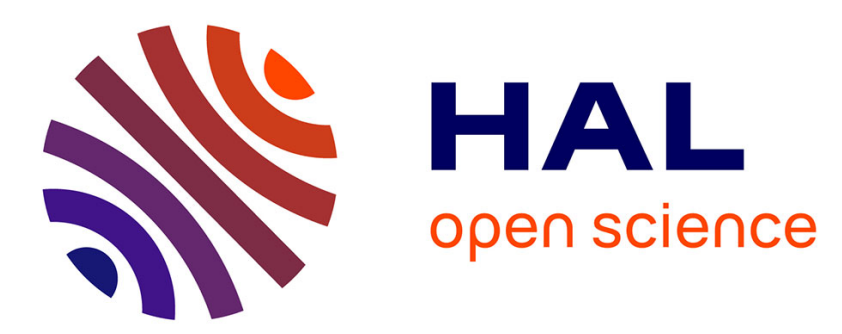

\title{
Numerical schemes for kinetic equations in the anomalous diffusion limit. Part II: degenerate collision frequency.
}

Nicolas Crouseilles, Hélène Hivert, Mohammed Lemou

\section{- To cite this version:}

Nicolas Crouseilles, Hélène Hivert, Mohammed Lemou. Numerical schemes for kinetic equations in the anomalous diffusion limit. Part II: degenerate collision frequency. . SIAM Journal on Scientific Computing, 2016, 38 (4). hal-01245312

\section{HAL Id: hal-01245312 \\ https://hal.inria.fr/hal-01245312}

Submitted on 17 Dec 2015

HAL is a multi-disciplinary open access archive for the deposit and dissemination of scientific research documents, whether they are published or not. The documents may come from teaching and research institutions in France or abroad, or from public or private research centers.
L'archive ouverte pluridisciplinaire HAL, est destinée au dépôt et à la diffusion de documents scientifiques de niveau recherche, publiés ou non, émanant des établissements d'enseignement et de recherche français ou étrangers, des laboratoires publics ou privés. 


\title{
NUMERICAL SCHEMES FOR KINETIC EQUATIONS IN THE ANOMALOUS DIFFUSION LIMIT. PART II: DEGENERATE COLLISION FREQUENCY.
}

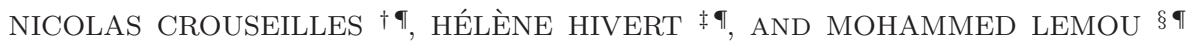

\begin{abstract}
In this work, which is the continuation of [9], we propose numerical schemes for linear kinetic equation which are able to deal with the fractional diffusion limit. When the collision frequency degenerates for small velocities it is known that for an appropriate time scale, the small mean free path limit leads to an anomalous diffusion equation. From a numerical point of view, this degeneracy gives rise to an additional stiffness that must be treated in a suitable way to avoid a prohibitive computational cost. Our aim is therefore to construct a class of numerical schemes which are able to undertake these stiffness. This means that the numerical schemes are able to capture the effect of small velocities in the small mean free path limit with a fixed set of numerical parameters. Various numerical tests are performed to illustrate the efficiency of our methods in this context.
\end{abstract}

Key words. BGK equation, Anomalous diffusion limit, Fractional diffusion equation, Asymptotic preserving schemes, Degenerate collision frequency.

AMS subject classifications. 35B25, 41A60, 65L04, 65M22.

1. Introduction. The theoretical and numerical study of particle system equations has major applications in plasma physics, galactic dynamics, chemotaxis or biological models. Depending on the physical properties of the system, different scales can be considered to describe it. When the mean free path of the particles is large compared to a typical length, a kinetic description of the system is required. Kinetic equations describe the time evolution of a distribution of particles depending on the time, the space and the velocity. This distribution of particles represents the probability for a particle to be at a given point of the phase space $(x, v)$ at the time $t$. When the mean free path of the particles is small, a macroscopic description can be used. In this regime, the former distribution function is close to an equilibrium and can be averaged in the velocity variable. The relevant quantity is then the density of the particles, which only depends on the time and the space.

The passage from the microscopic to the macroscopic scale is mathematically performed by asymptotic analysis. From a numerical point of view, the small mean free path limit corresponds to a singular perturbation problem which is known to be difficult to simulate (although a lot of works has already been achieved in this context). Indeed, this limit induces the presence of multiple scales in the system, so that the numerical parameters have to solve the smallest ones for stability reasons. As a consequence, the construction of efficient schemes in this context is of great interest.

In this work, we are interested in the time evolution of the distribution function $f$ which depends on the time $t>0$, the space variable $x \in \Omega \subset \mathbb{R}^{d}$ and the velocity $v \in V \subset \mathbb{R}^{d}$ with $d=1,2,3$. If $f$ stands for a distribution of particles, they undergo the effect of collisions which are modelized here by a linear operator $L$ acting on $f$ through

$$
L(f)(t, x, v)=\nu(v)\left(\rho_{\nu}(t, x) M(v)-f(t, x, v)\right),
$$

\footnotetext{
『IRMAR. Université de Rennes 1, Campus de Beaulieu. 35000 Rennes

${ }^{\dagger}$ INRIA-IPSO. Email: nicolas.crouseilles@inria.fr

¥Email: helene.hivert@univ-rennes1.fr

$\S$ CNRS. Email: mohammed.lemou@univ-rennes1.fr
} 
where

$$
\rho_{\nu}(t, x)=\frac{\langle\nu(v) f(t, x, v)\rangle_{V}}{\langle\nu(v) M(v)\rangle_{V}}=: \frac{\int_{V} \nu(v) f(t, x, v) \mathrm{d} v}{\int_{V} \nu(v) M(v) \mathrm{d} v}
$$

is not the usual density $\rho(t, x)=\langle f(t, x, v)\rangle_{V}$ but has been modified to ensure the mass conservation for $L:\langle L(f)\rangle_{V}=0$. In the sequel, we will always denote by brackets the integration over $v$. The equilibrium $M(v)$ will be taken constant on $V=\{v,|v| \leq \delta\}$ (we choose $\delta=1$ in the sequel)

$$
M(v)=M_{0},|v| \leq 1, \quad M_{0}>0,
$$

such that $\langle M(v)\rangle_{V}=1$ and we will consider the case of a degenerate collision frequency

$$
\nu(v)=\nu_{0}|v|^{d+2+\beta},|v| \leq 1
$$

for a given $\beta>0$. Hence, the second order moment of $M$ is not finite

$$
D=\int_{|v| \leq 1} \frac{v \otimes v}{\nu(v)} M(v) \mathrm{d} v=+\infty
$$

In order to capture a nontrivial asymptotic model, a suitable scaling has to be considered (see for instance [2]). We consider the kinetic equation

$$
\varepsilon^{\alpha} \partial_{t} f+\varepsilon v \cdot \nabla_{x} f=L(f), \text { with } \alpha=\frac{2 d+2+\beta}{d+1+\beta} \in(1,2),
$$

where $\varepsilon>0$ is the Knudsen number, which is proportional to the mean free path of the particles. Equation (1.5) has to be supplemented with an initial condition $f(0, x, v)=f_{0}(x, v)$ and spatial periodic boundary conditions are considered.

Considering $\alpha=2$, an equilibrium function $M$ and a collision frequency $\nu$ such that the integral (1.4) is finite, (1.5) degenerates into a diffusion equation for $\rho$

$$
\partial_{t} \rho(t, x)-\nabla_{x} \cdot\left(D \nabla_{x}\right) \rho(t, x)=0,
$$

when $\varepsilon$ goes to zero. There is a huge literature on this subject, we refer for example to $[18,27,4,10]$ for details. When the diffusion coefficient (1.4) is no longer finite, this asymptotic breaks down which can be interpreted by saying that the time scale $t \sim \varepsilon^{-2}$ is too long. Then, it is necessary to consider an appropriate scaling to get a nontrivial limit for (1.5). For instance, it is known (see [23, 21, 3]) that when the collision frequency is not degenerated for small velocities and when the equilibrium $M$ is an heavy-tailed function (equivalent to a power of $v$ for large velocities) (1.5) degenerates into a fractional diffusion equation when $\varepsilon$ goes to zero. As the scaling $\varepsilon^{\alpha}$ is not an integer power of $\varepsilon$ this fractional diffusion limit is called an anomalous diffusion limit. This scaling arises in the study of granular media (see $[12,6,5]$ ), plasmas (see [11]) or even economy ([17]). The case with degenerate collision frequency we are considering has been studied in [2]. It does not correspond to a particles dynamics physical situation but rather to the modelization of some chains of oscillators, see [13, 22].

When $\varepsilon$ goes to 0 , the solution of (1.5) converges to $\rho(t, x) M(v)$ where $\rho$ is the solution of the fractional diffusion equation that can be written in Fourier variable

$$
\partial_{t} \hat{\rho}(t, k)=-\kappa|k|^{\alpha} \hat{\rho}(t, k),
$$


where $\hat{\rho}$ stands for the space Fourier transform of $\rho, k$ is the Fourier variable and $\kappa$ is a constant which depends on $M, \nu$ and $\alpha$ and is defined by

$$
\kappa=\frac{M_{0} \nu_{0}^{1-\alpha}}{d+1+\beta}\left\langle\frac{1}{|v|^{d+\alpha}} \frac{(v \cdot e)^{2}}{1+(v \cdot e)^{2}}\right\rangle_{\mathbb{R}^{d}},
$$

for any $e \in \mathbb{R}^{d}$ such that $|e|=1$ (note that $\kappa$ does not depend on $e$ ). The fractional diffusion equation can also be written in the space variable

$$
\partial_{t} \rho(t, x)=-\frac{1}{c_{d, \alpha}} \frac{M_{0} \nu_{0}^{1-\alpha} \Gamma(\alpha+1)}{d+1+\beta}\left(-\Delta_{x}\right)^{\frac{\alpha}{2}} \rho(t, x),
$$

where $\Gamma$ is the usual Gamma function defined by $\Gamma(x)=\int_{0}^{+\infty} t^{x-1} \mathrm{e}^{-t} \mathrm{~d} t$ and $c_{d, \alpha}$ is a normalization constant given by $c_{d, \alpha}=\frac{\alpha \Gamma\left(\frac{d+\alpha}{2}\right)}{2 \pi^{\frac{d}{2}+\alpha} \Gamma\left(1-\frac{\alpha}{2}\right)}$. The fractional Laplacian $\left(-\Delta_{x}\right)^{\frac{\alpha}{2}}$ can be defined with its Fourier variable

$$
\left(\left(\widehat{\left.-\Delta_{x}\right)^{\frac{\alpha}{2}}} \rho\right)(k)=|k|^{\alpha} \hat{\rho}(k),\right.
$$

but has also an integral definition

$$
\left(-\Delta_{x}\right)^{\frac{\alpha}{2}} \rho(x)=c_{d, \alpha} P . V . \int_{\mathbb{R}^{d}} \frac{\rho(x+y)-\rho(x)}{|y|^{d+\alpha}} \mathrm{d} y,
$$

where P.V. is the principal value distribution.

From a numerical point of view, the presence of $\varepsilon$ makes the numerical simulations challenging. Indeed, if one uses a naive approach, a relation linking the space and time numerical parameters to the stiffness $\varepsilon$ (typically $\Delta t \sim \varepsilon^{\alpha}$, where $\Delta t$ is the time step) is required for stability reasons, which implies a redhibitory cost when $\varepsilon$ is small. The Asymptotic Preserving (AP) schemes are designed to overcome this strong restriction on the numerical parameters. Indeed, if we consider the continuous problem $P_{\varepsilon}$ degenerating into $P_{0}$ when $\varepsilon$ goes to zero, we ask the scheme $S_{\varepsilon}^{h}$ to be consistent with the problem $P_{\varepsilon}$ when the discretization parameter $h$ tends to 0 and to degenerate into a scheme $S_{0}^{h}$ solving the problem $P_{0}$ when $\varepsilon \rightarrow 0$. An AP scheme can also enjoy the stronger property of being Uniformly Accurate (UA). It means that the accuracy in $h$ of the scheme does not depend of $\varepsilon$. Note that it is very desirable for an AP scheme to enjoyy the stronger property of being Uniformly Accurate but this is not automatic and is a difficult issue in general. For kinetic equations, AP scheme has already been developed (see [14]) and there is an important literature for the diffusion asymptotic, for example $[15,25,24,19,20,16,7,1]$. In the case of a fractional diffusion scaling, the previous schemes do not enjoy the AP property. Indeed, as the velocities must be discretized with a finite number of points, the diffusion coefficient (1.4), which is an infinite integral in the case of the anomalous diffusion, appears to be always finite at the numerical level. Hence, the effects of the low velocities are missed and the approaches for the classical diffusion show only a numerical diffusion which does not match with the expected fractional diffusion.

In the case of the fractional diffusion induced by a heavy-tailed equilibrium, we set in a previous work ([9]) three numerical schemes enjoying the AP property. As the fractional diffusion limit did not naturally appear in the formulation of the schemes, we had to suitably modify them to make the fractional diffusion arise numerically. Let us remark that when $\alpha \in(1,2)$, an alternative approach has been proposed in 
[26] for the case of heavy tail equilibria. In this paper, we present an extension of the strategy presented in [9] when the fractional diffusion comes from the degeneracy of the collision frequency. In addition to the technical difficulties which are inherent to the velocity dependency of $\nu$ there are many important differences with the heavy-tail case that we have to face. First, to numerically capture the important effects of the low velocities (instead of large velocities in the heavy-tail case). Second, in the present case, the renormalized density $\rho_{\nu}$ defined in (1.1) is different from the usual density $\rho$ while these two quantities coincide in the heavy-tail case. This property induces an additional difficulty, as we will see, and a specific treatment is required in order to cover an asymptotic preserving property for the scheme. Lastly, the convergence rate to the fractional diffusion limit is very slow, so that the intermediate regime involves a large range of $\varepsilon$; as a consequence, the numerical validation turns out to be more difficult.

We will present three different Asymptotic Preserving schemes. First, we consider a fully implicit scheme for (1.5) in which Fourier space variable is considered. The delicate issue of the velocity discretization is highlighted to explain how the fractional diffusion arises. Then, a second scheme is proposed which allows to avoid, when desired, both the use of the space Fourier transform and that of a fully implicit scheme. A micro-macro formulation of (1.5) is then studied to write an semi-implicit scheme enjoying the AP property. Once again, the terms giving theoretically the anomalous diffusion limit will have to be treated with care to ensure the AP character of the scheme. Finally, a scheme based on a Duhamel formulation of (1.5) is proposed, which enjoys the stronger property of being uniformly accurate (UA) with respect to $\varepsilon$. These results were announced in [8]. Moreover, a proof of the convergence of the solution of (1.5) to the solution of (1.8) is given in [2]. We propose a formal derivation of the convergence rate in $\varepsilon$ for this limit. This convergence rate is illustrated in the numerical tests that we present in the last part.

The paper is organized as follows. In the next section, we will start by establishing a formal derivation of the fractional diffusion limit equation (1.8), and the rate of this convergence is formally derived (the related computation is postponed in the appendix). Then, in Section 3, we present the three asymptotic preserving schemes for (1.5). Eventually, we will present in the last section some numerical tests to highlight the properties of our schemes.

2. Formal derivation of the fractional diffusion limit. In this section, we formally derive the fractional diffusion (1.6) from the kinetic equation (1.5), where the computations are based on spatial Fourier transform. This will be the basis of the numerical methods proposed in Section 3. The proof has been done in [2].

We remind that $\hat{f}(t, k, v)$ (resp. $\hat{\rho}(t, k))$ denotes the spatial Fourier transform of $f(t, x, v)($ resp. $\rho(t, x))$

$$
\hat{f}(t, k, v)=\int_{\mathbb{R}^{d}} e^{-\mathrm{i} k \cdot x} f(t, x, v) \mathrm{d} x
$$

Proposition 2.1. Let $\hat{f}$ be the solution of (1.5) in the Fourier variable for a given initial condition $\hat{f}_{0}$. Then, when $\varepsilon \rightarrow 0, \hat{f}$ converges to $\hat{\rho} M$ with $\hat{\rho}$ solution of the fractional diffusion equation

$$
\partial_{t} \hat{\rho}(t, k)+\kappa|k|^{\alpha} \hat{\rho}(t, k)=0
$$

with $\kappa$ defined by (1.7) and with initial condition $\hat{\rho}(0, k)=\left\langle\hat{f}_{0}(k, v)\right\rangle_{V}$. 
Proof. Considering the Fourier transform (in space) of (1.5), we get

$$
\partial_{t} \hat{f}+\varepsilon^{-\alpha}(\nu+\mathrm{i} \varepsilon k \cdot v) \hat{f}=\varepsilon^{-\alpha} \nu \hat{\rho}_{\nu} M .
$$

Integrating with respect to the velocity variable leads to

$$
\partial_{t} \hat{\rho}+\mathrm{i} \varepsilon^{1-\alpha}\langle k \cdot v \hat{f}\rangle_{V}=0 .
$$

From (2.1), we can write

$$
\hat{f}=\frac{\nu \hat{\rho}_{\nu} M}{\nu+\mathrm{i} \varepsilon k \cdot v}-\varepsilon^{\alpha} \frac{\partial_{t} \hat{f}}{\nu+\mathrm{i} \varepsilon k \cdot v},
$$

so that $\hat{f} \underset{\varepsilon \rightarrow 0}{\longrightarrow} \hat{\rho} M$ and $(2.2)$ becomes

$$
\partial_{t} \hat{\rho}+\mathrm{i} \varepsilon^{1-\alpha}\left\langle\frac{\nu k \cdot v M}{\nu+\mathrm{i} \varepsilon k \cdot v}\right\rangle_{V} \hat{\rho}_{\nu}=O\left(\varepsilon^{\gamma}\right), \gamma>0 .
$$

The fact that the right hand side is negligible compared to the left hand side will be detailed in the Appendix. Then, the fractional diffusion operator is obtained by considering the limit $\varepsilon \rightarrow 0$ in the second term of the left hand side. Let us focus on this term in the sequel. First, since $M$ and $\nu$ are even, we write

$$
\mathrm{i} \varepsilon^{1-\alpha}\left\langle\frac{\nu k \cdot v M}{\nu+\mathrm{i} \varepsilon k \cdot v}\right\rangle_{V}=\varepsilon^{2-\alpha}\left\langle\frac{\nu(k \cdot v)^{2} M}{\nu^{2}+\varepsilon^{2}(k \cdot v)^{2}}\right\rangle_{V} .
$$

We can remark that the limit $\varepsilon \rightarrow 0$ is not defined since the term into brackets goes to infinity when $\varepsilon \rightarrow 0$ because of the degeneracy of $\nu$ for small velocities. Then, to capture the effect of small velocities, we perform the change of variable $w=\varepsilon|k| v / \nu$ to get

$$
\lim _{\varepsilon \rightarrow 0} \mathrm{i} \varepsilon^{1-\alpha}\left\langle\frac{\nu k \cdot v M}{\nu+\mathrm{i} \varepsilon k \cdot v}\right\rangle_{V}=\frac{M_{0} \nu_{0}^{1-\alpha}}{d+1+\beta}\left\langle\frac{1}{|w|^{d+\alpha}} \frac{(w \cdot e)^{2}}{1+(w \cdot e)^{2}}\right\rangle_{\mathbb{R}^{d}}|k|^{\alpha}=\kappa|k|^{\alpha}
$$

where $\kappa$ has been defined in (1.7). Moreover, since when $\varepsilon \rightarrow 0$, we have $\hat{\rho} \rightarrow \hat{\rho}_{\nu}$, we then deduce the fractional diffusion asymptotic model from (2.4).

Furthermore, we have the following result for the convergence rate (at the formal level). The proof is postponed to the Appendix.

Proposition 2.2. Let $\hat{f}$ be the solution of (1.5) in the Fourier variable for a given initial condition $\hat{f}_{0}$. We get the following rate of convergence

$$
\hat{f}-\hat{\rho} M=O\left(\varepsilon^{\frac{d}{d+1+\beta}}\right)+O\left(\varepsilon^{\frac{\beta}{d+1+\beta}}\right) .
$$

3. Numerical schemes. In this section, we present three different numerical schemes, based respectively on a fully implicit scheme, a micro-macro formulation and an integral formulation of (1.5), designed to approximate the solution of (1.5). We will denote $t_{n}=n \Delta t, 0 \leq n \leq N$ the time discretization, such that $N \Delta t=T$ and we will set $f^{n} \simeq f\left(t_{n}\right)$. The space domain $\Omega$ is bounded and we consider periodic conditions, allowing the use of the Fourier variable. At the discrete level, we will use the discrete Fourier transform. The computation of the numerical solution requires the 
use of a velocity discretization. In the tests, we will consider a first order quadrature method based on a uniform fixed set of $N_{v}$ points $v_{j}$, symmetrically distributed in the domain $|v| \leq 1$. In the sequel, we will denote by $\langle\cdot\rangle_{N_{v}, D}$ the discrete integration in $v$ on a domain $D$ :

$$
\langle f\rangle_{N_{v}, D}=\Delta v \sum_{\substack{j \in \mathbb{1}, N_{v} \rrbracket \\ v_{j} \in D}} f\left(v_{j}\right)
$$

We want these schemes to be Asymptotic Preserving (AP), indeed that for any initial condition $f_{0}$

(i) when $\varepsilon$ is fixed, the solution given by the numerical scheme is consistent with the solution of (1.5),

(ii) when the numerical parameters are fixed, the scheme degenerates into a scheme solving the anomalous diffusion equation (1.8) when $\varepsilon$ tends to 0 .

Since the fractional Laplacian of the asymptotic equation (1.6) comes from the small velocities of the degenerate collision frequency, it is necessary to take it into account in the schemes to ensure the AP property. One idea would be to consider an adaptive grid which is refined around $v=0$ but this will impose to link the velocity discretization to $\varepsilon$, which breaks down the AP property. We propose a method based on an analytical modification of the terms degenerating into the fractional Laplacian in the scheme. This modification consists into applying a change of variable in some integrals in $v$ of the scheme before discretizing them. Once it is done, we are able to show that the three schemes we propose preserve the asymptotic of fractional diffusion for a fixed set of numerical parameters.

3.1. Implicit scheme. The first idea to write a scheme for (1.5) which has the AP property is to use a fully implicit formulation of (1.5) in Fourier variable. It is well-known that in the case of the classical diffusion limit, such a scheme preserves the asymptotics, but here in the case of the fractional diffusion limit, it is not true, and a suitable modification of the scheme has to be performed to ensure the AP property.

We start with (1.5) written in Fourier variable and we consider a fully implicit time discretization

$$
\frac{\hat{f}^{n+1}-\hat{f}^{n}}{\Delta t}+\frac{\nu}{\varepsilon^{\alpha}}\left(1+\mathrm{i} \frac{\varepsilon k \cdot v}{\nu}\right) \hat{f}^{n+1}=\frac{\nu}{\varepsilon^{\alpha}} \hat{\rho}_{\nu}^{n+1} M,
$$

that is

$$
\hat{f}^{n+1}=\frac{1-\lambda(v)}{1+\mathrm{i} \lambda(v) \frac{\varepsilon k \cdot v}{\nu}} \hat{f}^{n}+\frac{\lambda(v)}{1+\mathrm{i} \lambda(v) \frac{\varepsilon k \cdot v}{\nu}} \hat{\rho}_{\nu}^{n+1} M,
$$

with $\lambda(v)=\Delta t \nu(v) /\left(\varepsilon^{\alpha}+\Delta t \nu(v)\right)$. Remark that $0 \leq \lambda(v) \leq 1, \lambda$ goes to 1 when $\varepsilon \rightarrow 0$ and $\lambda$ goes to 0 as $\Delta t \rightarrow 0$. So when $\varepsilon \rightarrow 0$ with fixed $\Delta t$, we get

$$
\hat{f}^{n+1}=\hat{\rho}_{\nu}^{n+1} M
$$

which is the expected limit for $\hat{f}$. Now, it remains to find an expression for $\hat{\rho}_{\nu}^{n+1}$. To do so, we multiply (3.2) by $\nu$ and integrate in $v$ to get

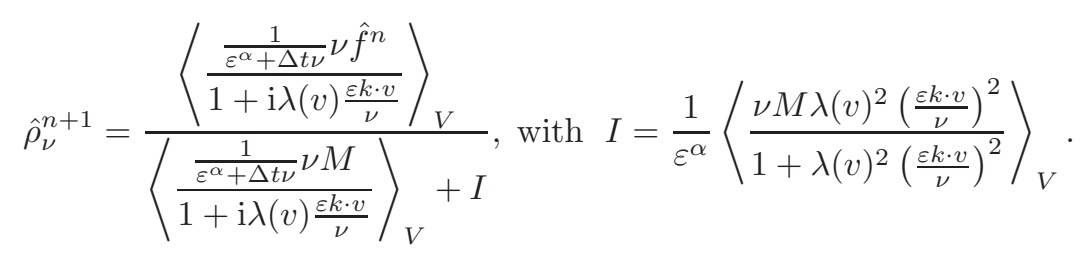


At the continuous level, the term $I$ gives the anomalous diffusion limit when $\varepsilon$ goes to zero. Indeed, the change of variable $w=\varepsilon|k| v / \nu(v)$ in $I$ enables to capture the small velocity effects so that $\lim _{\varepsilon \rightarrow 0} I=\kappa|k|^{\alpha}$, where $\kappa$ has been defined in (1.7). However, the situation is completely different at the numerical level, namely when a discretization in velocity with $N_{v}$ points has to be used. Indeed, a naive quadrature of $I$ given by (3.4) would lead to

$$
I \approx \varepsilon^{2-\alpha} \sum_{j=1}^{N_{v}} \frac{\nu\left(v_{j}\right) M\left(v_{j}\right) \Delta t^{2}\left(k \cdot v_{j}\right)^{2}}{\left(\varepsilon^{\alpha}+\Delta t \nu\left(v_{j}\right)\right)^{2}+\varepsilon^{2} \Delta t^{2}\left(k \cdot v_{j}\right)^{2}} \underset{\varepsilon \rightarrow 0}{\sim} \varepsilon^{2-\alpha} \sum_{j=1}^{N_{v}} \frac{\left(k \cdot v_{j}\right)^{2}}{\nu\left(v_{j}\right)} M\left(v_{j}\right) .
$$

This implies that $I$ goes to zero when $\varepsilon$ goes to zero since the velocity discretization is fixed (remember that $\alpha<2$ ). Therefore, the asymptotic numerical scheme becomes $\hat{\rho}_{\nu}^{n+1}=\hat{\rho}_{\nu}^{n}$ when $\varepsilon \rightarrow 0$, which is not a consistent numerical scheme for the asymptotic model. To recover the correct asymptotic behavior, the main idea is to transform the expression of $I$ at the continuous level before applying the discretization in order to make the anomalous diffusion operator appear in it. To do so, we perform a change of variables $w=\varepsilon|k| v / \nu(v)$ in $I$ to get

$$
\begin{aligned}
& w=\varepsilon|k| v / \nu(v) \text { in } I \text { to get } \\
& I=\frac{|k|^{\alpha} \nu_{0}^{1-\alpha} M_{0}}{d+1+\beta} \int_{|w| \geq \frac{\varepsilon|k|}{\nu_{0}}} \frac{1}{\mid w^{d+\alpha}} \frac{\left(\frac{\Delta t \varphi}{\varepsilon^{\alpha}+\Delta t \varphi}\right)^{2}(w \cdot e)^{2}}{1+\left(\frac{\Delta t \varphi}{\varepsilon^{\alpha}+\Delta t \varphi}\right)^{2}(w \cdot e)^{2}} \mathrm{~d} w,
\end{aligned}
$$

where $e$ denotes any unitary vector and $\varphi=\nu_{0}\left(\varepsilon|k| /\left(\nu_{0}|w|\right)\right)^{\frac{d+2+\beta}{d+1+\beta}}$. Note that $I$ does not depend on the choice of $e$. Once the change of variables has been done, the velocity discretization can be performed so that we get

$$
I \approx \frac{|k|^{\alpha} \nu_{0}^{1-\alpha} M_{0}}{d+1+\beta}\left\langle\frac{1}{|w|^{d+\alpha}} \frac{\left(\frac{\Delta t \varphi}{\varepsilon^{\alpha}+\Delta t \varphi}\right)^{2}(w \cdot e)^{2}}{1+\left(\frac{\Delta t \varphi}{\varepsilon^{\alpha}+\Delta t \varphi}\right)^{2}(w \cdot e)^{2}}\right\rangle_{N_{v},\left|w_{\max }\right| \geq|w| \geq \frac{\varepsilon|k|}{\nu_{0}}}
$$

where $\langle\cdot\rangle_{N_{v}, D}$ has been defined in (3.1). Let us remark that when $\varepsilon$ goes to zero, this approximation of $I$ degenerates towards

$$
\frac{|k|^{\alpha} \nu_{0}^{1-\alpha} M_{0}}{d+1+\beta}\left\langle\frac{1}{|w|^{d+\alpha}} \frac{(w \cdot e)^{2}}{1+(w \cdot e)^{2}}\right\rangle_{N_{v},|w| \leq\left|w_{\max }\right|}=: \bar{\kappa}
$$

which is a consistent approximation of $\kappa$ given by (1.7). Let us remark that the numerical integration is done up to a maximum discrete velocity $\left|w_{\max }\right|$. Eventually, we have the following proposition

Proposition 3.1. We consider the following scheme defined for all $k$ and for all time indices $0 \leq n \leq N, N \Delta t=T$ by

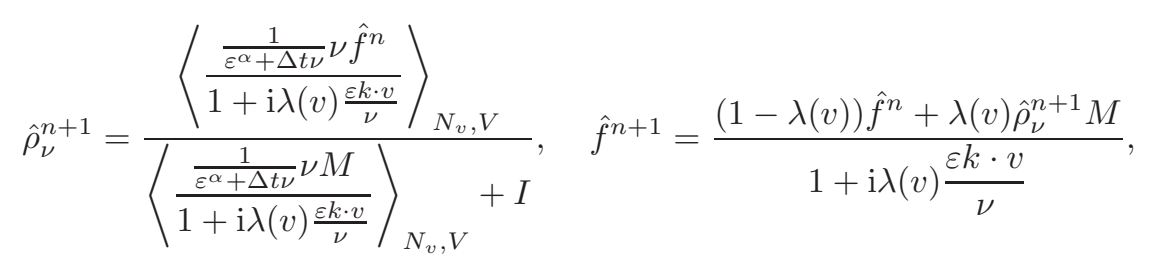

with I given by (3.5), $\hat{\rho}^{n+1}=\left\langle\hat{f}^{n+1}\right\rangle_{N_{v}, V}, \lambda(v)=\Delta t \nu(v) /\left(\varepsilon^{\alpha}+\Delta t \nu(v)\right)$ and $e$ is any unitary vector and with the initial condition $\hat{f}^{0}(k, v)=\hat{f}_{0}(k, v)$. This scheme has the following properties: 
(i) The scheme is of order 1 for any fixed $\varepsilon$ and preserves the total mass.

(ii) The scheme is AP: for a fixed $\Delta t$, the scheme solves the anomalous diffusion equation when $\varepsilon$ goes to zero

$$
\frac{\hat{\rho}^{n+1}(k)-\hat{\rho}^{n}(k)}{\Delta t}=-\bar{\kappa}|k|^{\alpha} \hat{\rho}^{n+1}(k) \text {, with } \bar{\kappa} \text { given by }(3.6) \text {. }
$$

REMARK 1. We can go further, modifying the approximation (3.5) of I by

$$
I \approx \frac{\Delta t^{2}}{\left(\varepsilon^{\alpha-\frac{d+2+\beta}{d+1+\beta}}+\Delta t\right)^{2}} \bar{\kappa}
$$

where $\bar{\kappa}$ is defined in (3.6). The so-obtained scheme enjoys the same properties stated in the above proposition.

Proof. The first point of (i) is a direct consequence of the use of a fully implicit Euler scheme for (1.5). For the conservation of the total mass, let us write (3.7) for $k=0$ and integrate with respect to $v$

$$
\begin{aligned}
& \left\langle\frac{\nu M}{\varepsilon^{\alpha}+\Delta t \nu}\right\rangle_{N_{v}, V} \hat{\rho}_{\nu}^{n+1}(0)=\left\langle\frac{\nu}{\varepsilon^{\alpha}+\Delta t \nu} \hat{f}^{n}(0)\right\rangle_{N_{v}, V} \\
& \hat{\rho}^{n+1}(0)=\hat{\rho}^{n}(0)-\Delta t\left\langle\frac{\nu}{\varepsilon^{\alpha}+\Delta t \nu} \hat{f}^{n}(0)\right\rangle_{N_{v}, V}+\left\langle\frac{\Delta t \nu}{\varepsilon^{\alpha}+\Delta t \nu} M\right\rangle_{N_{v}, V} \hat{\rho}_{\nu}^{n+1}(0)
\end{aligned}
$$

Injecting (3.9) in (3.10) leads to the result.

For the AP character of the scheme (ii), letting $\varepsilon$ to 0 with fixed $\Delta t$ in the first equation of (3.7) leads to

$$
\frac{\hat{\rho}_{\nu}^{n+1}-\hat{\rho}^{n}}{\Delta t}=-\bar{\kappa}|k|^{\alpha} \hat{\rho}_{\nu}^{n+1}
$$

and with (3.3) we have $\hat{\rho}^{n}=\hat{\rho}_{\nu}^{n}, \forall n \geq 1$ when $\varepsilon \rightarrow 0$. It implies that (3.7) degenerates into (3.8).

3.2. Micro-macro decomposition based scheme. In the previous part, we presented a fully implicit scheme designed to solve (1.5); however the use of Fourier transform may be restrictive in some cases and one may have to use the original space variable. Therefore, making the transport part implicit in time may induce high computational cost especially in the multidimensional case. Here we write a scheme based on a micro-macro decomposition of the distribution function $f$ in which the transport part is explicit. As for the previous scheme, suitable modifications have to be done to ensure the AP character.

Denoting $f(t, x, v)=\rho(t, x) M(v)+g(t, x, v)$ such that $\langle g\rangle_{V}=0$, we insert this decomposition into (1.5) before integrating in $v$ to get

$$
\partial_{t} \rho+\varepsilon^{1-\alpha}\left\langle v \cdot \nabla_{x} g\right\rangle_{V}=0
$$

Then we multiply this last equality by $M$ and subtract it from (1.5) to write an expression for $g$

$$
\partial_{t} g+\varepsilon^{1-\alpha} v \cdot \nabla_{x} \rho M+\varepsilon^{1-\alpha}\left(v \cdot \nabla_{x} g-\left\langle v \cdot \nabla_{x} g\right\rangle_{V} M\right)=-\frac{1}{\varepsilon^{\alpha}} \nu\left(g-\frac{\langle\nu g\rangle_{V} M}{\langle\nu M\rangle_{V}}\right) .
$$


From (3.11) and (3.12) we can write the following semi-discrete micro-macro scheme (see $[20,19])$

$$
\left\{\begin{aligned}
\frac{\rho^{n+1}-\rho^{n}}{\Delta t}+ & \varepsilon^{1-\alpha}\left\langle v \cdot \nabla_{x} g^{n+1}\right\rangle_{V}=0 \\
\frac{g^{n+1}-g^{n}}{\Delta t}+ & \varepsilon^{1-\alpha} v \cdot \nabla_{x} \rho^{n} M+\varepsilon^{1-\alpha}\left(v \cdot \nabla_{x} g^{n}-\left\langle v \cdot \nabla_{x} g^{n}\right\rangle_{V} M\right) \\
& =-\frac{1}{\varepsilon^{\alpha}} \nu\left(g^{n+1}-\frac{\left\langle\nu g^{n+1}\right\rangle_{V}}{\langle\nu M\rangle_{V}} M\right)
\end{aligned}\right.
$$

As previously, if the velocity integrations are done directly using the approximation $\langle\cdot\rangle_{V} \approx\langle\cdot\rangle_{N_{v}, V}$ where the discrete bracket $\langle\cdot\rangle_{N_{v}, V}$ is given by (3.1), the so-obtained scheme does not enjoy the AP property. Indeed, as $g^{n+1}$ goes to zero when $\varepsilon$ goes to zero, we have

$$
\varepsilon v \cdot \nabla_{x} \rho^{n} M=-\nu\left(g^{n+1}-\frac{\left\langle\nu g^{n+1}\right\rangle_{N_{v}, V}}{\langle\nu M\rangle_{N_{v}, V}} M\right)+o(\varepsilon)
$$

that is, for nonzero $v$

$$
g^{n+1}=\frac{\left\langle\nu g^{n+1}\right\rangle_{N_{v}, V}}{\langle\nu M\rangle_{N_{v}, V}} M-\frac{\varepsilon}{\nu} v \cdot \nabla_{x} \rho^{n} M+o(\varepsilon)
$$

Then, we insert this expression in the first line of (3.13), reminding that $M$ is even, to get

$$
\frac{\rho^{n+1}-\rho^{n}}{\Delta t}-\varepsilon^{2-\alpha}\left\langle\frac{M}{\nu} v \cdot \nabla_{x}\left(v \cdot \nabla_{x} \rho^{n}\right)\right\rangle_{N_{v}, V}=o(\varepsilon) .
$$

As in the previous part, the term into brackets is finite although it is not finite at the continuous level. Hence, when $\varepsilon \rightarrow 0$, (3.13) degenerates into $\rho^{n+1}=\rho^{n}$ which of course does not solve the fractional diffusion equation. To obtain the right asymptotic scheme, we must modify (3.13) to make the anomalous diffusion limit appear. To do so, we first express the term $\left\langle v \cdot \nabla_{x} g^{n+1}\right\rangle_{V}=\left\langle v \cdot \nabla_{x} f^{n+1}\right\rangle_{V}$ appearing in the first line of (3.13) with a semi-discrete implicit formulation of (1.5)

$$
f^{n+1}=\lambda(v)\left(I+\varepsilon \frac{\lambda(v)}{\nu} v \cdot \nabla_{x}\right)^{-1} \rho_{\nu}^{n+1} M+(1-\lambda(v))\left(I+\varepsilon \frac{\lambda(v)}{\nu} v \cdot \nabla_{x}\right)^{-1} f^{n}
$$

with $\lambda(v)=\Delta t \nu(v) /\left(\varepsilon^{\alpha}+\Delta t \nu(v)\right)$. As $\varepsilon \lambda(v) / \nu(v)=O(\Delta t)$ we will rather inject the following approximation for $f^{n+1}$ in (3.13) (recalling that $v \in V$ )

$$
f^{n+1}=\lambda(v)\left(I+\varepsilon \frac{\lambda(v)}{\nu} v \cdot \nabla_{x}\right)^{-1} \rho_{\nu}^{n+1} M+(1-\lambda(v))\left(I-\varepsilon \frac{\lambda(v)}{\nu} v \cdot \nabla_{x}\right) f^{n} .
$$

Indeed, since the scheme is first order accurate, it is possible to remove terms of order $\Delta t^{2}$ with no incidence on its global accuracy. Hence, we decide to keep only the ones which play a role in the AP character of the scheme. With additional simplifications, the first line of (3.13) becomes

$$
\begin{aligned}
\frac{\rho^{n+1}-\rho^{n}}{\Delta t} & +\frac{1}{\varepsilon^{\alpha}}\left\langle\varepsilon \lambda(v) v \cdot \nabla_{x}\left(I+\frac{\varepsilon \lambda(v)}{\nu} v \cdot \nabla_{x}\right)^{-1} \rho_{\nu}^{n+1} M\right\rangle_{V} \\
& +\varepsilon^{1-\alpha}\left\langle(1-\lambda(v)) v \cdot \nabla_{x} g^{n}\right\rangle_{V}=0,
\end{aligned}
$$


because

$$
\left\langle(1-\lambda(v)) v \cdot \nabla_{x}\left(I-\frac{\varepsilon \lambda(v)}{\nu} v \cdot \nabla_{x}\right) f^{n}\right\rangle_{V}=\left\langle(1-\lambda(v)) v \cdot \nabla_{x} g^{n}\right\rangle_{V}+O(\Delta t) .
$$

As the anomalous diffusion limit comes from the second term of (3.14), we have to rewrite it in order to make the fractional Laplacian appear. First, we have to express $\rho_{\nu}$ in terms of $\rho$; to do that, we inject the decomposition $f=\rho M+g$ in the definition of $\rho_{\nu}$

$$
\rho_{\nu}=\frac{\langle\nu f\rangle_{V}}{\langle\nu M\rangle_{V}}=\frac{\langle\nu(\rho M+g)\rangle_{V}}{\langle\nu M\rangle_{V}}=\rho+\frac{\langle\nu g\rangle_{V}}{\langle\nu M\rangle_{V}} .
$$

Then, using space Fourier transform, we rewrite (3.14) as

$$
\frac{\rho^{n+1}-\rho^{n}}{\Delta t}+\mathcal{F}^{-1}\left(\mathcal{I} \hat{\rho}^{n+1}\right)+\mathcal{F}^{-1}\left(\mathcal{I} \frac{\left\langle\nu \hat{g}^{n+1}\right\rangle_{V}}{\langle\nu M\rangle_{V}}\right)+\varepsilon^{1-\alpha}\left\langle(1-\lambda(v)) v \cdot \nabla_{x} g^{n}\right\rangle_{V}=0,
$$

where $\mathcal{F}^{-1}$ denotes the inverse Fourier transform and

$$
\mathcal{I}=\varepsilon^{1-\alpha}\left\langle\frac{\mathrm{i} \lambda(v) k \cdot v M(v)}{1+\mathrm{i} \frac{\varepsilon \lambda(v)}{\nu} k \cdot v}\right\rangle_{V}=\varepsilon^{-\alpha}\left\langle\frac{\lambda(v)^{2} \frac{(\varepsilon k \cdot v)^{2}}{\nu} M}{1+\lambda(v)^{2}\left(\frac{\varepsilon k \cdot v}{\nu}\right)^{2}}\right\rangle_{V} .
$$

Let us remark that for $k=0$, we have $\mathcal{I}=0$. For $k \neq 0$, the change of variables $w=\varepsilon|k| v / \nu(v)$ is performed in $\mathcal{I}$ before applying the discretization $\langle\cdot\rangle_{N_{v}, V}$ (using the definitions (1.2) and (1.3) of $M$ and $\nu$ ). Then, we have

$$
\mathcal{I} \approx \frac{|k|^{\alpha} \nu_{0}^{1-\alpha} M_{0}}{d+1+\beta}\left\langle\frac{1}{|w|^{d+\alpha}} \frac{(w \cdot e)^{2}}{(w \cdot e)^{2}+(1+\psi)^{2}}\right\rangle_{N_{v},\left|w_{\max }\right| \geq|w| \geq \frac{\varepsilon|k|}{\nu_{0}}}
$$

where $\psi=\frac{\varepsilon^{q}}{\Delta t} \nu_{0}^{r}(|w| /|k|)^{p}, p=\frac{d+2+\beta}{d+1+\beta}$ and $q=\frac{d}{d+1+\beta}, r=\frac{1}{d+1+\beta}$.

To compute $\left(\rho^{n+1}, g^{n+1}\right)$ recursively with the second line of (3.13) and (3.15), we need to determine $\left\langle\nu g^{n+1}\right\rangle_{N_{v}, V}$. To do that, we start with the with the second line of (3.13) which gives an expression for $g^{n+1}$

$$
g^{n+1}=\frac{g^{n}-\frac{\Delta t}{\varepsilon^{\alpha}} \mathcal{T}^{n}}{1+\frac{\Delta t}{\varepsilon^{\alpha}} \nu}+\frac{\Delta t \nu M}{\langle\nu M\rangle_{N_{v}}\left(\varepsilon^{\alpha}+\Delta t \nu\right)}\left\langle\nu g^{n+1}\right\rangle_{N_{v}, V}
$$

with

$$
\mathcal{T}^{n}=\varepsilon v \cdot \nabla_{x} \rho^{n} M+\varepsilon\left(v \cdot \nabla_{x} g^{n}-\left\langle v \cdot \nabla_{x} g^{n}\right\rangle_{N_{v}, V} M\right) .
$$

Multiplying the expression for $g^{n+1}$ by $\nu$ and integrating it using $\langle\cdot\rangle_{N_{v}, V}$, we find an expression for $\left\langle\nu g^{n+1}\right\rangle_{N_{v}, V}$

$$
\left\langle\nu g^{n+1}\right\rangle_{N_{v}, V}=\frac{\left\langle\frac{\nu g^{n}}{1+\frac{\Delta t}{\varepsilon^{\alpha}} \nu(v)}\right\rangle_{N_{v}, V}-\frac{\Delta t}{\varepsilon^{\alpha}}\left\langle\frac{\nu \mathcal{T}^{n}}{1+\frac{\Delta t}{\varepsilon^{\alpha}} \nu}\right\rangle_{N_{v}, V}}{1-\frac{\Delta t}{\varepsilon^{\alpha}\langle\nu M\rangle_{N_{v}, V}}\left\langle\frac{\nu^{2} M}{1+\frac{\Delta t}{\varepsilon^{\alpha}} \nu}\right\rangle_{N_{v}, V}}
$$

Finally, we have the following proposition. 
Proposition 3.2. We consider the following scheme (discretized in time and velocity) defined for all $x \in \mathbb{R}^{d}, v \in V$ and all time indices $0 \leq n \leq N$ with $N \Delta t=$ $T(T>0)$, by

$$
\left\{\begin{array}{c}
\left\langle\nu g^{n+1}\right\rangle_{N_{v}, V}=\langle\nu M\rangle_{N_{v}, V} \frac{\left\langle\frac{\nu g^{n}+\mathcal{T}^{n}}{\varepsilon^{\alpha}+\Delta t \nu}\right\rangle_{N_{v}, V}}{\left\langle\frac{\nu^{2} M}{\varepsilon^{\alpha}+\Delta t \nu}\right\rangle_{N_{v}, V}} \\
\frac{g^{n+1}-g^{n}}{\Delta t}+\mathcal{T}^{n}=-\frac{1}{\varepsilon^{\alpha}} \nu\left(g^{n+1}-\frac{\left\langle\nu g^{n+1}\right\rangle_{N_{v}, V}}{\langle\nu M\rangle_{N_{v}, V}} M\right) \\
\frac{\rho^{n+1}-\rho^{n}}{\Delta t}+\mathcal{F}^{-1}\left(\mathcal{I} \hat{\rho}^{n+1 / 2}\right)+\mathcal{F}^{-1}\left(\mathcal{I} \frac{\left\langle\nu \hat{g}^{n+1}\right\rangle_{N_{v}, V}}{\langle\nu M\rangle_{N_{v}, V}}\right) \\
+\varepsilon^{1-\alpha}\left\langle(1-\lambda(v)) v \cdot \nabla_{x} g^{n}\right\rangle_{N_{v}, V}=0,
\end{array}\right.
$$

with $\mathcal{T}^{n}$ defined in (3.17), $\lambda(v)=\Delta t \nu(v) /\left(\varepsilon^{\alpha}+\Delta t \nu(v)\right)$, where $\mathcal{F}^{-1}$ denotes the inverse Fourier transform in space and with the initial conditions $\rho^{0}(x)=\rho(0, x)=$ $\left\langle f_{0}(x, v)\right\rangle_{N_{v}, V}$ and $g^{0}(x, v)=f_{0}(x, v)-\rho(0, x) M(v)$. The quantity $\hat{\rho}^{n+1 / 2}$ can be chosen equal to $\hat{\rho}^{n}$ or to $\hat{\rho}^{n+1}$ depending on the desired asymptotic scheme (explicit or implicit in time) and $\mathcal{I}$ is given by (3.16). This scheme has the following properties:

(i) The scheme is of order 1 for any fixed $\varepsilon$ and preserves the total mass.

(ii) The scheme is AP: for a fixed $\Delta t$, it solves the anomalous diffusion equation when $\varepsilon$ goes to zero

$$
\frac{\hat{\rho}^{n+1}(k)-\hat{\rho}^{n}(k)}{\Delta t}=-\bar{\kappa}|k|^{\alpha} \hat{\rho}^{n+1 / 2}(k),
$$

where $\bar{\kappa}$ is defined by (3.6).

REMARK 2. Assuming that the space and velocity discretizations have been fixed, $\left(\rho^{n}, g^{n}\right)$ are computed recursively with (3.18). The first line of the scheme enables to compute $\left\langle\nu g^{n+1}\right\rangle_{N_{v}, V}$ which is reported in the second and third lines to compute $g^{n+1}$ and $\rho^{n+1}$.

As the term $\mathcal{I}$ defined in (3.16) has been precomputed to make the anomalous diffusion operator clearly appear, the velocity discretization is not a difficult issue anymore. Once it is defined, the integrals in $v$ are computed with any quadrature method, such as rectangle formula. Finally, a standard upwind scheme is used to approximate the spatial derivative appearing in the term $\mathcal{T}^{n}$ defined in (3.17).

REMARK 3. To guarantee the structure of the micro-macro model, it is important that the scheme satisfies $\left\langle g^{n+1}\right\rangle_{N_{v}, V}=0$. To do that, $g^{n+1}$ is modified consistently as $g^{n+1} \leftarrow g^{n+1}-\left\langle g^{n+1}\right\rangle_{N_{v}, V} M$.

REMARK 4. As in the fully implicit scheme, we can go further by approximating consistently $\mathcal{I}$ given by (3.16) as

$$
\mathcal{I}=\frac{\Delta t^{2}}{\Delta t^{2}+\varepsilon^{q}\left(\Delta t+\varepsilon^{q}\right)}|k|^{\alpha} \bar{\kappa},
$$

where $\bar{\kappa}$ is given by (3.6) and $q=d /(d+1+\beta)$. The so-obtained micro-macro based scheme still enjoys the same properties stated in the above proposition.

Proof. The proof of (i) is immediate from the derivation of the scheme, let us prove (ii). At first, we remark that $\mathcal{T}^{n}$ goes to 0 as $\varepsilon \rightarrow 0$, then the first line of (3.18) 
gives that $\left\langle\nu(v) g^{n+1}\right\rangle_{N_{v}, V}$ goes to 0 as $\varepsilon \rightarrow 0$ because $\left\langle g^{n}\right\rangle_{N_{v}}=0$. After that, the second line of (3.18) implies that $g^{n+1}=o(1)$. When reported in the fourth line of (3.18), as $\varepsilon^{1-\alpha}(1-\lambda(v))=O(\varepsilon)$, we get

$$
\frac{\rho^{n+1}-\rho^{n}}{\Delta t}+\mathcal{F}^{-1}\left(\mathcal{I} \hat{\rho}^{n+1 / 2}\right)=o(1)
$$

Finally, $\mathcal{I}$ is equivalent to $\bar{\kappa}|k|^{\alpha}$ for small $\varepsilon$, with $\bar{\kappa}$ given by (3.6).

3.3. Scheme based on an integral formulation of the equation (1.5). In the previous parts, we wrote two AP schemes solving (1.5). In this section, in the spirit of [9], we present a scheme based on an integral formulation of (1.5). However, the presence of the collision frequency $\nu$ requires specific developments to ensure the AP property. In particular, for general initial data, the strategy proposed in [9] leads to an $\mathrm{AP}$ scheme for $\rho_{\nu}$ instead of $\rho$ (which are different if $f$ is not at equilibrium). Then, the present section is decomposed into two parts: first, the case of equilibrium initial data is tackled, for which the scheme is proved to be uniformly accurate, and second the more delicate case of general initial data is studied, for which the scheme is proved to be AP.

3.3.1. Equilibrium initial data. We start from the Duhamel form for $\hat{f}$

$$
\hat{f}(t, k, v)=\mathrm{e}^{-\frac{t}{\varepsilon^{\alpha}}(\nu+\mathrm{i} \varepsilon k \cdot v)} \hat{f}_{0}(k, v)+\nu M \int_{0}^{\frac{t}{\varepsilon^{\alpha}}} \mathrm{e}^{-s(\nu+\mathrm{i} \varepsilon k \cdot v)} \hat{\rho}_{\nu}\left(t-\varepsilon^{\alpha} s, k\right) \mathrm{d} s,
$$

which we multiply by $\nu$, integrate in $v$ and evaluate at $t=t^{n+1}$ to get

$$
K \hat{\rho}_{\nu}\left(t^{n+1}, k\right)=\hat{A}_{0}\left(t^{n+1}, k\right)+\int_{0}^{\frac{t^{n+1}}{\varepsilon^{\alpha}}}\left\langle\nu^{2} M \mathrm{e}^{-s(\nu+\mathrm{i} \varepsilon k \cdot v)}\right\rangle_{V} \hat{\rho}_{\nu}\left(t^{n+1}-\varepsilon^{\alpha} s, k\right) \mathrm{d} s,
$$

where

$$
K=\langle\nu M\rangle_{V}, \text { and } \hat{A}_{0}(t, k)=\left\langle\nu \mathrm{e}^{-\frac{t}{\varepsilon^{\alpha}}(\nu+\mathrm{i} \varepsilon k \cdot v)} \hat{f}_{0}(k, v)\right\rangle_{V} .
$$

Then, we get

$$
K \hat{\rho}_{\nu}\left(t_{n+1}, k\right)=\sum_{j=0}^{n} \int_{\frac{t_{j}}{\varepsilon^{\alpha}}}^{\frac{t_{j+1}}{\varepsilon^{\alpha}}}\left\langle\nu^{2} M \mathrm{e}^{-s(\nu+\mathrm{i} \varepsilon k \cdot v)}\right\rangle_{V} \hat{\rho}_{\nu}\left(t_{n+1}-\varepsilon^{\alpha} s, k\right) \mathrm{d} s+\hat{A}_{0}\left(t_{n+1}, k\right),
$$

where $\hat{A}_{0}$ and $K$ are defined in (3.21). We perform a quadrature of order 2 in the integrals in $s$. Assuming that the time derivatives of $\hat{\rho}_{\nu}$ are uniformly bounded in $\varepsilon$, we have $\forall j \in \llbracket 0, N-1 \rrbracket, \forall s \in\left[\frac{t_{j}}{\varepsilon^{\alpha}}, \frac{t_{j+1}}{\varepsilon^{\alpha}}\right]$,

$$
\hat{\rho}_{\nu}\left(t_{n+1}-\varepsilon^{\alpha} s, k\right)=\left(1-\frac{\varepsilon^{\alpha} s-t_{j}}{\Delta t}\right) \hat{\rho}_{\nu}\left(t_{n+1-j}, k\right)+\frac{\varepsilon^{\alpha} s-t_{j}}{\Delta t} \hat{\rho}_{\nu}\left(t_{n-j}, k\right)+O\left(\Delta t^{2}\right) .
$$

Inserting this expression in the integral terms of (3.22) leads to

$$
\begin{aligned}
\int_{\frac{t_{j}}{\varepsilon^{\alpha}}}^{\frac{t_{j+1}}{\varepsilon^{\alpha}}}\left\langle\nu^{2} M \mathrm{e}^{-s(\nu+\mathrm{i} \varepsilon k \cdot v)}\right\rangle_{V} & \hat{\rho}_{\nu}\left(t_{n+1}-\varepsilon^{\alpha} s, k\right) \mathrm{d} s=c_{j}(\varepsilon, k) \hat{\rho}_{\nu}\left(t_{n+1}-t_{j}, k\right) \\
& +b_{j}(\varepsilon, k) \hat{\rho}_{\nu}\left(t_{n+1}-t_{j+1}, k\right)+O\left(\Delta t^{2}\right),
\end{aligned}
$$


where the remainder is bounded independently of $\varepsilon$ and where we denoted

$$
\begin{aligned}
& b_{j}(\varepsilon, k)=\int_{\frac{t_{j}}{\varepsilon^{\alpha}}}^{\frac{t_{j+1}}{\varepsilon^{\alpha}}} \frac{\varepsilon^{\alpha} s-t_{j}}{\Delta t}\left\langle\nu^{2} M \mathrm{e}^{-s(\nu+\mathrm{i} \varepsilon k \cdot v)}\right\rangle_{V} \mathrm{~d} s, \\
& c_{j}(\varepsilon, k)=\int_{\frac{t_{j}}{\varepsilon^{\alpha}}}^{\frac{t_{j+1}}{\varepsilon^{\alpha}}}\left(1-\frac{\varepsilon^{\alpha} s-t_{j}}{\Delta t}\right)\left\langle\nu^{2} M \mathrm{e}^{-s(\nu+\mathrm{i} \varepsilon k \cdot v)}\right\rangle_{V} \mathrm{~d} s .
\end{aligned}
$$

Then, we use the quadrature (3.24) in (3.22) to write (using the obvious notations $\left.b_{j}:=b_{j}(\varepsilon, k)\right)$ and $\left.c_{j}:=c_{j}(\varepsilon, k)\right)$

$$
\hat{\rho}_{\nu}\left(t_{n+1}, k\right)=\frac{1}{K} \sum_{j=0}^{n}\left[c_{j} \hat{\rho}_{\nu}\left(t_{n+1-j}, k\right)+b_{j} \hat{\rho}_{\nu}\left(t_{n-j}, k\right)+O\left(\Delta t^{2}\right)\right]+\frac{1}{K} \hat{A}_{0}\left(t_{n+1}, k\right) .
$$

As $N \Delta t=T$, then $n \Delta t^{2} \leq T \Delta t$ and the last expression permits to write a first order scheme for $\hat{\rho}_{\nu}$ that writes

$$
\hat{\rho}_{\nu}^{n+1}(k)=\frac{\hat{A}_{0}\left(t_{n+1}, k\right)+\sum_{j=1}^{n}\left(c_{j} \hat{\rho}_{\nu}^{n+1-j}(k)+b_{j} \hat{\rho}_{\nu}^{n-j}(k)\right)+b_{0} \hat{\rho}_{\nu}^{n}(k)}{K-c_{0}} .
$$

To ensure the AP property for this scheme, the discretization of the terms $b_{j}$ and $c_{j}$ is crucial: as in the previous cases, their limit as $\varepsilon$ goes to zero makes the anomalous diffusion operator appear, but when computed numerically it does not arise because of the effects of the low velocities missed with the discretization. Indeed, we consider the velocity integration appearing in (3.25)-(3.26)

$$
\left\langle\nu^{2} M \mathrm{e}^{-s(\nu+\mathrm{i} \varepsilon k \cdot v)}\right\rangle_{V}=\left\langle\nu^{2} M\left(\mathrm{e}^{-s(\nu+\mathrm{i} \varepsilon k \cdot v)}-\mathrm{e}^{-\nu(v) s}\right)\right\rangle_{V}+\left\langle\nu^{2} M \mathrm{e}^{-\nu(v) s}\right\rangle_{V} .
$$

At the continuous level, the first term in the right hand side gives the anomalous diffusion when $\varepsilon$ goes to zero. Indeed, with $M$ and $\nu$ defined by (1.2) and (1.3), the change of variables $w=\varepsilon|k| v / \nu$ in the integral in $v$ reads

$$
\begin{aligned}
& \left\langle\nu^{2} M\left(\mathrm{e}^{-s(\nu+\mathrm{i} \varepsilon k \cdot v)}-\mathrm{e}^{-\nu(v) s}\right)\right\rangle_{V} \\
& =\frac{\varepsilon^{\alpha}|k|^{\alpha} \nu_{0}^{1-\alpha} M_{0}}{d+1+\beta}\left\langle\varphi \frac{1}{|w|^{d+\alpha}}\left(\mathrm{e}^{-\varphi s(1+\mathrm{i} w \cdot e)}-\mathrm{e}^{-s \varphi}\right)\right\rangle_{|w| \geq \frac{\varepsilon|k|}{\nu_{0}}},
\end{aligned}
$$

with $\varphi=\nu_{0}\left(\frac{\varepsilon|k|}{\nu_{0}|w|}\right)^{\frac{d+2+\beta}{d+1+\beta}}$ and where $e$ denotes any unitary vector. Here, the fractional diffusion limit clearly appears. We then inject (3.29) computed with discrete brackets $\langle\cdot\rangle_{N_{v},\left|w_{\max }\right| \geq|w| \geq \varepsilon|k| / \nu_{0}}$ in (3.28) and eventually compute explicitly the integrals in $s$ 
of (3.25)-(3.26) to get the following expression of $b_{j}$ and $c_{j}$

$$
\begin{aligned}
& b_{j}(\varepsilon, k)=\frac{\varepsilon^{\alpha}}{\Delta t}\left\langle M \mathrm{e}^{-\frac{t_{j}}{\varepsilon^{\alpha}} \nu}\left(1-\mathrm{e}^{-\frac{\Delta t}{\varepsilon^{\alpha}} \nu}\right)\right\rangle_{N_{v}, V}-\left\langle\nu M \mathrm{e}^{-\frac{t_{j+1}}{\varepsilon^{\alpha}} \nu}\right\rangle_{N_{v}, V} \\
& +\frac{\varepsilon^{\alpha}|k|^{\alpha} \nu_{0}^{1-\alpha} M_{0}}{d+1+\beta}\left\langle\frac { 1 } { | w | ^ { d + \alpha } } \left[\mathrm{e}^{-\frac{t_{j+1}}{\varepsilon^{\alpha}} \varphi}-\frac{\mathrm{e}^{-\frac{t_{j+1}}{\varepsilon^{\alpha}} \varphi(1+\mathrm{i} w \cdot e)}}{1+\mathrm{i} w \cdot e}\right.\right. \\
& \left.+\frac{\varepsilon^{\alpha}}{\Delta t}\left(\frac{\mathrm{e}^{-\frac{t_{j}}{\varepsilon^{\alpha}} \varphi(1+\mathrm{i} w \cdot e)}\left(1-\mathrm{e}^{-\frac{\Delta t}{\varepsilon^{\alpha}} \varphi(1+\mathrm{i} w \cdot e)}\right)}{\varphi(1+\mathrm{i} w \cdot e)^{2}}-\frac{\mathrm{e}^{-\frac{t_{j}}{\varepsilon^{\alpha}} \varphi}\left(1-\mathrm{e}^{-\frac{\Delta t}{\varepsilon^{\alpha}} \varphi}\right)}{\varphi}\right)\right\rangle_{N_{v}, \mathcal{W}} \\
& c_{j}(\varepsilon, k)=\left\langle\nu M \mathrm{e}^{-\frac{t_{j}}{\varepsilon^{\alpha}} \nu}\right\rangle_{N_{v}, V}-\frac{\varepsilon^{\alpha}}{\Delta t}\left\langle M \mathrm{e}^{-\frac{t_{j}}{\varepsilon^{\alpha}} \nu}\left(1-\mathrm{e}^{-\frac{\Delta t}{\varepsilon^{\alpha}} \nu}\right)\right\rangle_{N_{v}, V} \\
& +\frac{\varepsilon^{\alpha}|k|^{\alpha} \nu_{0}^{1-\alpha} M_{0}}{d+1+\beta}\left\langle\frac { 1 } { | w | ^ { d + \alpha } } \left[\frac{\mathrm{e}^{-\frac{t_{j}}{\varepsilon^{\alpha}} \varphi(1+\mathrm{i} w \cdot e)}}{1+\mathrm{i} w \cdot e}-\mathrm{e}^{-\frac{t_{j}}{\varepsilon^{\alpha}} \varphi}\right.\right. \\
& \left.-\frac{\varepsilon^{\alpha}}{\Delta t}\left(\frac{\mathrm{e}^{-\frac{t_{j}}{\varepsilon^{\alpha}} \varphi(1+\mathrm{i} w \cdot e)}\left(1-\mathrm{e}^{-\frac{\Delta t}{\varepsilon^{\alpha}} \varphi(1+\mathrm{i} w \cdot e)}\right)}{\varphi(1+\mathrm{i} w \cdot e)^{2}}\right)\right\rangle_{N_{v}, \mathcal{W}},
\end{aligned}
$$

with $\mathcal{W}=\left\{w \in \mathbb{R}^{d},\left|w_{\max }\right| \geq|w| \geq \varepsilon|k| / \nu_{0}\right\}$. The so-obtained scheme (3.27) for $\hat{\rho}_{\nu}$ where $b_{j}, c_{j}$ are given by (3.30)-(3.31) enables us to find a way to recover the distribution function $\hat{f}$ in Fourier variable. Indeed, with a Duhamel formulation of (1.5) integrated between $t_{n}$ and $t_{n+1}$ it is possible to write an expression for $\hat{f}\left(t_{n+1}, k, v\right)$

$$
\hat{f}\left(t_{n+1}, k, v\right)=\mathrm{e}^{-\frac{\Delta t}{\varepsilon^{\alpha}}(\nu+\mathrm{i} \varepsilon k \cdot v)} \hat{f}\left(t_{n}, k, v\right)+\int_{0}^{\frac{\Delta t}{\varepsilon^{\alpha}}} \mathrm{e}^{-s(\nu+\mathrm{i} \varepsilon k \cdot v)} \hat{\rho}_{\nu}\left(t_{n+1}-\varepsilon^{\alpha} s, k\right) \mathrm{d} s \nu M .
$$

Then, the quadrature (3.23) leads to

$$
\hat{f}\left(t_{n+1}, k, v\right)=\mathrm{e}^{-\frac{\Delta t}{\varepsilon^{\alpha}}(\nu+\mathrm{i} \varepsilon k \cdot v)} \hat{f}\left(t_{n}, k, v\right)+\nu M\left(\gamma \hat{\rho}_{\nu}\left(t_{n+1}, k\right)+\beta \hat{\rho}_{\nu}\left(t_{n}, k\right)\right)+O\left(\Delta t^{2}\right),
$$

where

$$
\gamma=\int_{0}^{\frac{\Delta t}{\varepsilon^{\alpha}}}\left(1-\frac{\varepsilon^{\alpha} s}{\Delta t}\right) \mathrm{e}^{-s(\nu+\mathrm{i} \varepsilon k \cdot v)} \mathrm{d} s, \quad \beta=\int_{0}^{\frac{\Delta t}{\varepsilon^{\alpha}}} \frac{\varepsilon^{\alpha} s}{\Delta t} \mathrm{e}^{-s(\nu+\mathrm{i} \varepsilon k \cdot v)} \mathrm{d} s .
$$

This provides a first order scheme for $\hat{f}$ using the values $\hat{\rho}_{\nu}^{n}$ determined with (3.27)

$$
\hat{f}^{n+1}=\mathrm{e}^{-\frac{\Delta t}{\varepsilon x}(\nu+\mathrm{i} \varepsilon k \cdot v)} \hat{f}^{n}+\nu M\left(\gamma \hat{\rho}_{\nu}^{n+1}+\beta \hat{\rho}_{\nu}^{n}\right),
$$

with the values of $\beta$ and $\gamma$ computed exactly to ensure the AP property of the scheme

$$
\gamma=\frac{1}{\nu+\mathrm{i} \varepsilon k \cdot v}-\frac{\varepsilon^{\alpha}}{\Delta t} \frac{1-\mathrm{e}^{-\frac{\Delta t}{\varepsilon^{\alpha}}(\nu+\mathrm{i} \varepsilon k \cdot v)}}{(\nu+\mathrm{i} \varepsilon k \cdot v)^{2}}, \quad \beta=\frac{\varepsilon^{\alpha}}{\Delta t} \frac{1-\mathrm{e}^{-\frac{\Delta t}{\varepsilon^{\alpha}}(\nu+\mathrm{i} \varepsilon k \cdot v)}}{(\nu+\mathrm{i} \varepsilon k \cdot v)^{2}}-\frac{\mathrm{e}^{-\frac{\Delta t}{\varepsilon^{\alpha}}(\nu+\mathrm{i} \varepsilon k \cdot v)}}{\nu+\mathrm{i} \varepsilon k \cdot v} .
$$

A simple integration of $\hat{f}^{n+1}$ with $\langle\cdot\rangle_{N_{v}, V}$ gives an expression for $\hat{\rho}^{n+1}$. Eventually, we have the following proposition. 
Proposition 3.3. We consider the following scheme defined for all $k$ and for all time index $0 \leq n \leq N, N \Delta t=T$ by

$$
\left\{\begin{array}{l}
\hat{\rho}_{\nu}^{n+1}(k)=\frac{\hat{A}_{0}\left(t_{n+1}, k\right)+\sum_{j=1}^{n}\left(c_{j} \hat{\rho}_{\nu}^{n+1-j}(k)+b_{j} \hat{\rho}_{\nu}^{n-j}(k)\right)+b_{0} \hat{\rho}_{\nu}^{n}(k)}{K-c_{0}} \\
\hat{f}^{n+1}=\mathrm{e}^{-\frac{\Delta t}{\varepsilon^{\alpha}}(\nu+\mathrm{i} \varepsilon k \cdot v)} \hat{f}^{n}+\nu M\left(\gamma \hat{\rho}_{\nu}^{n+1}+\beta \hat{\rho}_{\nu}^{n}\right),
\end{array}\right.
$$

with $K, \hat{A}_{0}, b_{j}, c_{j}, \gamma$ and $\beta$ defined in (3.21)-(3.30)-(3.31) and (3.34). For an initial data $\hat{f}_{0}(k, v)$ at equilibrium, this scheme enjoys the following properties:

(i) The scheme is first order in time and preserves the total mass.

(ii) The scheme is AP: for a fixed $\Delta t$, it solves the anomalous diffusion equation when $\varepsilon$ goes to zero

$$
\frac{\hat{\rho}^{n+1}(k)-\hat{\rho}^{n}(k)}{\Delta t}=-\bar{\kappa}|k|^{\alpha} \hat{\rho}^{n+1}(k)
$$

where $\hat{\rho}^{n}=\left\langle\hat{f}^{n}\right\rangle_{N v, V}$ and $\bar{\kappa}$ is defined by (3.6).

(iii) Moreover, the semi-discrete-in-time scheme enjoys the UA property: it is first order uniformly in $\varepsilon$

$$
\exists C>0, \sup _{\varepsilon \in(0,1]}\left\|\hat{f}^{N}(\cdot, \cdot)-\hat{f}(T, \cdot, \cdot)\right\|_{L_{k, v}^{\infty}} \leq C \Delta t .
$$

REMARK 5. The numerical tests (see Fig. 4.3) suggests that this scheme is of order 2 for a fixed value of $\varepsilon$ and of order 1 uniformly in $\varepsilon$.

Proof. For the mass conservation (i), we integrate (at the discrete level) with respect to $v$ the second equation of (3.35) and evaluate it at $k=0$. For an initial data at equilibrium, we have $\hat{\rho}_{\nu}^{0}(k=0)=\hat{\rho}^{0}(k=0)$ so that

$$
\hat{\rho}^{1}=\left\langle e^{-\Delta t / \varepsilon^{\alpha} \nu} M\right\rangle_{N_{v}, V} \hat{\rho}^{0}+\langle\nu M \gamma\rangle_{N_{v}, V} \hat{\rho}_{\nu}^{1}+\langle\nu M \beta\rangle_{N_{v}, V} \hat{\rho}_{\nu}^{0}, \text { for } k=0 .
$$

From the first equation of (3.35), we easily get $\hat{\rho}_{\nu}^{1}(k=0)=\hat{\rho}^{0}(k=0)$ so that the previous equation leads to $\hat{\rho}^{1}(k=0)=\hat{\rho}^{0}(k=0)$. The mass conservation is deduced by induction, assuming that $\hat{\rho}_{\nu}^{\ell}(k=0)=\hat{\rho}^{\ell}(k=0)$ for $\ell=0, \ldots, n$.

For the AP character (ii), let us remark that with (3.30) and (3.31), the formula for $\hat{\rho}_{\nu}$ in (3.35) degenerates into

$$
\frac{\hat{\rho}_{\nu}^{n+1}(k)-\hat{\rho}_{\nu}^{n}(k)}{\Delta t}=-\bar{\kappa}|k|^{\alpha} \hat{\rho}_{\nu}^{n+1}(k) .
$$

Moreover, the second line of (3.35) gives $\hat{f}^{n+1}=\hat{\rho}_{\nu}^{n+1} M(v)$ for $n \geq 0$ when $\varepsilon \rightarrow 0$, that implies $\hat{\rho}^{n+1}=\hat{\rho}_{\nu}^{n+1}$ for small $\varepsilon$. As the initial condition is at equilibrium, we also have $\hat{\rho}^{0}=\hat{\rho}_{\nu}^{0}$. Eventually, when $\varepsilon \rightarrow 0$, (3.35) degenerates into

$$
\frac{\hat{\rho}^{n+1}(k)-\hat{\rho}^{n}(k)}{\Delta t}=-\bar{\kappa}|k|^{\alpha} \hat{\rho}^{n+1}(k),
$$

for all $n \leq N$, which proves the AP property. 
Now, let us prove that the semi-discrete (in time) scheme is first order accurate uniformly in $\varepsilon$ (iii). Using the notations (3.30)-(3.31), (3.22) writes

$$
\hat{\rho}_{\nu}\left(t_{n+1}, k\right)=\frac{1}{K} \hat{A}_{0}\left(t_{n+1}, k\right)+\frac{1}{K} \sum_{j=0}^{n} c_{j} \hat{\rho}_{\nu}\left(t_{n+1-j}, k\right)+b_{j} \hat{\rho}_{\nu}\left(t_{n-j}, k\right)+\frac{\Delta t^{2}}{K} F,
$$

where the remainder $F$ is given by

$$
\begin{aligned}
F & =-\frac{1}{2} \sum_{j=0}^{n} \int_{\frac{t_{j}}{\varepsilon^{\alpha}}}^{\frac{t_{j+1}}{\varepsilon^{\alpha}}}\left(\left(1-\frac{\varepsilon^{\alpha} s-t_{j}}{\Delta t}\right)\left(\frac{\varepsilon^{\alpha} s-t_{j}}{\Delta t}\right)^{2} \partial_{t}^{2} \hat{\rho}_{\nu}\left(t_{n+1}-\varepsilon^{\alpha} \xi_{1}(s), k\right)\right. \\
& \left.+\frac{\varepsilon^{\alpha} s-t_{j}}{\Delta t}\left(\frac{t_{j+1}-\varepsilon^{\alpha} s}{\Delta t}\right)^{2} \partial_{t}^{2} \hat{\rho}_{\nu}\left(t_{n+1}-\varepsilon^{\alpha} \xi_{2}(s), k\right)\right)\left\langle\nu^{2} M \mathrm{e}^{-s(\nu+\mathrm{i} \varepsilon k \cdot v)}\right\rangle_{V} \mathrm{~d} s
\end{aligned}
$$

with $\xi_{1}(s), \xi_{2}(s) \in\left(0, \Delta t / \varepsilon^{\alpha}\right)$. As $1 \geq\left(1-\frac{\varepsilon^{\alpha} s-t_{j}}{\Delta t}\right) \geq 0$ and $1 \geq \frac{\varepsilon^{\alpha} s-t_{j}}{\Delta t} \geq 0$ for $s \in\left[\frac{t_{j}}{\varepsilon^{\alpha}}, \frac{t_{j+1}}{\varepsilon^{\alpha}}\right]$, we have

$$
\begin{aligned}
|F| \leq & \frac{\left\|\partial_{t}^{2} \hat{\rho}_{\nu}\right\|_{\infty}}{2} \sum_{j=0}^{n} \int_{\frac{t_{j}}{\varepsilon^{\alpha}}}^{\frac{t_{j+1}}{\varepsilon^{\alpha}}}\left(\left(1-\frac{\varepsilon^{\alpha} s-t_{j}}{\Delta t}\right)\left(\frac{\varepsilon^{\alpha} s-t_{j}}{\Delta t}\right)^{2}\right. \\
& \left.+\frac{\varepsilon^{\alpha} s-t_{j}}{\Delta t}\left(\frac{t_{j+1}-\varepsilon^{\alpha} s}{\Delta t}\right)^{2}\right)\left\langle\nu^{2} M \mathrm{e}^{-s \nu}\right\rangle_{V} \mathrm{~d} s \\
\leq & \frac{\left\|\partial_{t}^{2} \hat{\rho}_{\nu}\right\|_{\infty}}{2} \sum_{j=0}^{n} \int_{\frac{t_{j}}{\varepsilon^{\alpha}}}^{\frac{t_{j+1}}{\varepsilon^{\alpha}}}\left(\frac{\varepsilon^{\alpha} s-t_{j}}{\Delta t}\right)\left\langle\nu^{2} M \mathrm{e}^{-s \nu}\right\rangle_{V} \mathrm{~d} s .
\end{aligned}
$$

Now, let us denote $E_{n}=\hat{\rho}_{\nu}\left(t_{n}, k\right)-\hat{\rho}_{\nu}^{n}(k)$ and suppose that $E_{0}=0$. From (3.27) and (3.36) we have

$$
\left(1-\frac{c_{0}}{K}\right) E_{n+1}=\frac{b_{0}}{K} E_{n}+\frac{1}{K} \sum_{j=1}^{n}\left(c_{j} E_{n+1-j}+b_{j} E_{n-j}\right)+\frac{\Delta t^{2}}{K} F .
$$

In the sequel, to get an estimate for $E_{n+1}$, we will need two different bounds for $\left(1-\frac{c_{0}}{K}\right)^{-1}=K /\left(K-c_{0}\right)$. From the definition (3.26) of $c_{0}$, we directly get

$$
\left|c_{0}\right| \leq \int_{0}^{\frac{\Delta t}{\varepsilon^{\alpha}}}\left(1-\frac{\varepsilon^{\alpha} s}{\Delta t}\right)\left\langle\nu^{2} M \mathrm{e}^{-s \nu}\right\rangle_{V} \mathrm{~d} s
$$

from which we deduce the first estimate for $\left|K /\left(K-c_{0}\right)\right|$

$$
\left|\frac{K}{K-c_{0}}\right| \leq \frac{K}{\langle\nu M\rangle_{V}-\int_{0}^{\frac{\Delta t}{\varepsilon^{\alpha}}}\left(1-\frac{\varepsilon^{\alpha} s}{\Delta t}\right)\left\langle\nu^{2} M \mathrm{e}^{-s \nu}\right\rangle_{V} \mathrm{~d} s} .
$$

Now, using $0 \leq 1-\frac{\varepsilon^{\alpha} s}{\Delta t} \leq 1$ for $s \in\left[0, \frac{\Delta t}{\varepsilon^{\alpha}}\right]$, we get

$$
\left|c_{0}\right| \leq\left\langle\nu^{2} M \int_{0}^{\frac{\Delta t}{\varepsilon \alpha}} \mathrm{e}^{-s \nu} \mathrm{d} s\right\rangle_{V} \leq K-\left\langle\nu M \mathrm{e}^{-\frac{\Delta t}{\varepsilon^{\alpha}} \nu}\right\rangle_{V}
$$


so that we deduce the second estimate for $\left|K /\left(K-c_{0}\right)\right|$

$$
\left|\frac{K}{K-c_{0}}\right| \leq K\left\langle\nu M \mathrm{e}^{-\frac{\Delta t}{\varepsilon^{\alpha}} \nu}\right\rangle_{V}^{-1} .
$$

In (3.39) let us focus on the term containing F. From (3.38), we have

$$
\begin{aligned}
\left|\frac{\frac{1}{K} F}{1-\frac{c_{0}}{K}}\right|=\left|\frac{F}{K-c_{0}}\right| & \leq \frac{\left\|\partial_{t}^{2} \hat{\rho}_{\nu}\right\|_{\infty}}{2} \frac{\int_{0}^{\frac{\Delta t}{\varepsilon^{\alpha}}}\left(\frac{\varepsilon^{\alpha} s}{\Delta t}\right)\left\langle\nu^{2} M \mathrm{e}^{-s \nu}\right\rangle_{V} \mathrm{~d} s}{\left|K-c_{0}\right|} \\
& +\frac{\left\|\partial_{t}^{2} \hat{\rho}_{\nu}\right\|_{\infty}}{2} \frac{\sum_{j=1}^{n} \int_{\frac{t_{j}}{\varepsilon^{\alpha}}}^{\frac{t_{j+1}}{\varepsilon^{\alpha}}}\left(\frac{\varepsilon^{\alpha} s-t_{j}}{\Delta t}\right)\left\langle\nu^{2} M \mathrm{e}^{-s \nu}\right\rangle_{V} \mathrm{~d} s}{\left|K-c_{0}\right|} \\
& =: \frac{\left\|\partial_{t}^{2} \hat{\rho}_{\nu}\right\|_{\infty}}{2}\left(T_{1}+T_{2}\right) .
\end{aligned}
$$

To find an estimate for $T_{1}$, we use (3.40) to write

$$
T_{1} \leq \frac{\int_{0}^{\frac{\Delta t}{\varepsilon^{\alpha}}}\left\langle\nu^{2} M \mathrm{e}^{-s \nu(v)}\right\rangle_{V} \mathrm{~d} s-\int_{0}^{\frac{\Delta t}{\varepsilon^{\alpha}}}\left(1-\frac{\varepsilon^{\alpha} s}{\Delta t}\right)\left\langle\nu^{2} M \mathrm{e}^{-s \nu}\right\rangle_{V} \mathrm{~d} s}{K-\int_{0}^{\frac{\Delta t}{\varepsilon^{\alpha}}}\left(1-\frac{\varepsilon^{\alpha} s}{\Delta t}\right)\left\langle\nu^{2} M \mathrm{e}^{-s \nu}\right\rangle_{V} \mathrm{~d} s},
$$

that implies that the first line of (3.42) is bounded by $\left\|\partial_{t}^{2} \hat{\rho}_{\nu}\right\|_{\infty} / 2$. Now, using (3.41), $T_{2}$ can be estimated as

$$
T_{2} \leq \int_{\frac{\Delta t}{\varepsilon^{\alpha}}}^{\frac{t_{n+1}}{\varepsilon^{\alpha}}}\left\langle\nu^{2} M \mathrm{e}^{-s \nu}\right\rangle_{V} \mathrm{~d} s\left|K-c_{0}\right|^{-1} \leq 1
$$

Eventually, (3.43) and (3.44) give

$$
\left|\frac{F}{K-c_{0}}\right| \leq\left\|\partial_{t}^{2} \hat{\rho}_{\nu}\right\|_{\infty}
$$

Now, for the other terms in (3.39), we introduce $\mathcal{E}_{n}=\max _{\substack{j=0 \ldots n \\ \varepsilon \in(0,1]}}\left|E_{j}\right|$. From the definition (3.30) and (3.31), we have for the first two terms of the right hand side of (3.39)

$$
\begin{aligned}
& \frac{1}{K}\left|b_{0} E_{n}+\sum_{j=1}^{n}\left(c_{j} E_{n+1-j}+b_{j} E_{n-j}\right)\right| \\
& \leq \frac{1}{K} \int_{0}^{\frac{\Delta t}{\varepsilon^{\alpha}}} \frac{\varepsilon^{\alpha} s}{\Delta t}\left|\left\langle\nu^{2} M \mathrm{e}^{-s(\nu+\mathrm{i} \varepsilon k \cdot v)}\right\rangle_{V} E_{n}\right| \mathrm{d} s \\
& +\sum_{j=1}^{n}\left(\int_{\frac{t_{j}}{\varepsilon^{\alpha}}}^{\frac{t_{j}+1}{\varepsilon^{\alpha}}}\left(1-\frac{\varepsilon^{\alpha} s-t_{j}}{\Delta t}\right)\left|\left\langle\nu^{2} M \mathrm{e}^{-s(\nu+\mathrm{i} \varepsilon k \cdot v)}\right\rangle_{V} E_{n+1-j}\right| \mathrm{d} s\right. \\
& \left.+\int_{\frac{t_{j}}{\varepsilon^{\alpha}}}^{\frac{t_{j+1}}{\varepsilon^{\alpha}}} \frac{\varepsilon^{\alpha} s-t_{j}}{\Delta t}\left|\left\langle\nu^{2} M \mathrm{e}^{-s(\nu+\mathrm{i} \varepsilon k \cdot v)}\right\rangle_{V} E_{n-j}\right| \mathrm{d} s\right) \\
& \leq \frac{1}{K}\left(K-\int_{0}^{\frac{\Delta t}{\varepsilon^{\alpha}}}\left(1-\frac{\varepsilon^{\alpha} s}{\Delta t}\right)\left\langle\nu^{2} M \mathrm{e}^{-\nu s}\right\rangle_{V} \mathrm{~d} s\right) \mathcal{E}_{n}
\end{aligned}
$$


Hence, using (3.40), we get

$$
\left|b_{0} E_{n}+\sum_{j=1}^{n}\left(c_{j} E_{n+1-j}+b_{j} E_{n-j}\right)\right|\left|K-c_{0}\right|^{-1} \leq \mathcal{E}_{n} .
$$

Eventually (3.39) gives, using (3.45) and (3.46), $E_{n+1} \leq \mathcal{E}_{n}+\left\|\partial_{t}^{2} \hat{\rho}_{\nu}\right\|_{\infty} \Delta t^{2}$. Hence $\mathcal{E}_{n+1} \leq \mathcal{E}_{n}+\left\|\partial_{t}^{2} \hat{\rho}_{\nu}\right\|_{\infty} \Delta t^{2}$. As we supposed $\mathcal{E}_{0}=0$, it writes

$$
\mathcal{E}_{n+1} \leq\left\|\partial_{t}^{2} \hat{\rho}_{\nu}\right\|_{\infty} T \Delta t
$$

where $T=N \Delta t$ is the final time. This proves that the scheme in $\hat{\rho}_{\nu}$ is first order accurate uniformly in $\varepsilon$.

Now, to prove that the scheme in $\hat{f}$ is first order uniformly in $\varepsilon$ we consider the Duhamel formulation for $\hat{f}$

$$
\hat{f}\left(t_{n+1}, k, v\right)=\mathrm{e}^{-\frac{\Delta t}{\varepsilon^{\alpha}}(\nu+\mathrm{i} \varepsilon k \cdot v)} \hat{f}\left(t_{n}, k, v\right)+\nu M \int_{0}^{\frac{\Delta t}{\varepsilon^{\alpha}}} \mathrm{e}^{-s(\nu+\mathrm{i} \varepsilon k \cdot v)} \hat{\rho}_{\nu}\left(t_{n+1}-\varepsilon^{\alpha} s, k\right) \mathrm{d} s,
$$

and with the same Taylor expansion as previously, we have

$$
\hat{f}\left(t_{n+1}, k, v\right)=\nu M\left(\gamma \hat{\rho}_{\nu}\left(t_{n+1}, k\right)+\beta \hat{\rho}_{\nu}\left(t_{n}, k\right)\right)+\nu M \Delta t^{2} G+\mathrm{e}^{-\frac{\Delta t}{\varepsilon^{\alpha}}(\nu+\mathrm{i} \varepsilon k \cdot v)} \hat{f}\left(t_{n}, k, v\right),
$$

with $\beta$ and $\gamma$ defined in (3.33) and

$$
\begin{aligned}
G & =-\frac{1}{2} \int_{0}^{\frac{\Delta t}{\varepsilon^{\alpha}}}\left[\left(1-\frac{\varepsilon^{\alpha} s}{\Delta t}\right)\left(\frac{\varepsilon^{\alpha} s}{\Delta t}\right)^{2} \partial_{t}^{2} \hat{\rho}_{\nu}\left(t_{n+1}-\varepsilon^{\alpha} \xi_{1}(s), k\right)\right. \\
& \left.+\left(\frac{\varepsilon^{\alpha} s}{\Delta t}\right)\left(1-\frac{\varepsilon^{\alpha} s}{\Delta t}\right)^{2} \partial_{t}^{2} \hat{\rho}_{\nu}\left(t_{n+1}-\varepsilon^{\alpha} \xi_{2}(s), k\right)\right] \mathrm{e}^{-s(\nu+\mathrm{i} \varepsilon k \cdot v)} \mathrm{d} s
\end{aligned}
$$

where $\xi_{1}(s), \xi_{2}(s) \in\left(0, \Delta t / \varepsilon^{\alpha}\right)$. As previously, we can estimate $|\nu M G|$

$$
\begin{gathered}
|\nu \quad M G| \leq \nu M \frac{\left\|\partial_{t}^{2} \hat{\rho}_{\nu}\right\|_{\infty}}{2} \int_{0}^{\frac{\Delta t}{\varepsilon^{\alpha}}}\left[\left(1-\frac{\varepsilon^{\alpha} s-t_{j}}{\Delta t}\right)\left(\frac{\varepsilon^{\alpha} s-t_{j}}{\Delta t}\right)^{2}\right. \\
\left.+\frac{\varepsilon^{\alpha} s-t_{j}}{\Delta t}\left(\frac{\varepsilon^{\alpha} s-t_{j+1}}{\Delta t}\right)^{2}\right]\left|\mathrm{e}^{-s(\nu+\mathrm{i} \varepsilon k \cdot v)}\right| \mathrm{d} s \\
\leq \nu M \frac{\left\|\partial_{t}^{2} \hat{\rho}_{\nu}\right\|_{\infty}}{2} \int_{0}^{\frac{\Delta t}{\varepsilon^{\alpha}}} \mathrm{e}^{-s \nu} \mathrm{d} s \leq C\left(1-\mathrm{e}^{-\frac{\Delta t}{\varepsilon^{\alpha}} \nu}\right) .
\end{gathered}
$$

One can now look at the truncation error $E_{n}^{f}=\hat{f}\left(t_{n}, k, v\right)-\hat{f}^{n}(k, v)$ which satisfies

$$
E_{n+1}^{f}=\mathrm{e}^{-\frac{\Delta t}{\varepsilon^{\alpha}}(\nu+\mathrm{i} \varepsilon k \cdot v)} E_{n}^{f}+\nu M\left(\gamma E_{n+1}+\beta E_{n}\right)+\nu M \Delta t^{2} G .
$$

The term $\nu M\left(\gamma E_{n+1}+\beta E_{n}\right)$ can be estimated by (using definition (3.33))

$$
\left|\nu M\left(\gamma E^{n+1}+\beta E^{n}\right)\right| \leq \nu M \int_{0}^{\frac{\Delta t}{\varepsilon^{\alpha}}} \mathrm{e}^{-s \nu} \mathrm{d} s \mathcal{E}_{n+1} \leq C\left(1-\mathrm{e}^{-\frac{\Delta t}{\varepsilon^{\alpha}} \nu}\right) \Delta t
$$


where we used (3.47) for the last inequality. Hence, denoting $\Lambda_{n}=\max _{v \neq 0, k, \varepsilon \in(0,1]}\left|E_{n}^{f}\right|$ we get from (3.49): $\Lambda_{n+1} \leq \mathrm{e}^{-\frac{\Delta t}{\varepsilon^{\alpha}} \nu} \Lambda_{n}+C\left(1-\mathrm{e}^{-\frac{\Delta t}{\varepsilon^{\alpha}} \nu}\right)\left(\Delta t^{2}+\Delta t\right)$. With $\Lambda_{0}=0$, it finally comes that

$$
\Lambda_{n+1} \leq C\left(1-\mathrm{e}^{-\frac{\Delta t}{\varepsilon^{\alpha}} \nu}\right)\left(\Delta t+\Delta t^{2}\right) \sum_{j=0}^{n} \mathrm{e}^{-j \frac{\Delta t}{\varepsilon^{\alpha}} \nu} \leq C \Delta t
$$

3.3.2. General initial data. In the previous subsection, we observed that the asymptotic scheme of (3.35) as $\varepsilon \rightarrow 0$ writes

$$
\frac{\hat{\rho}_{\nu}^{n+1}(k)-\hat{\rho}_{\nu}^{n}(k)}{\Delta t}=-\bar{\kappa}|k|^{\alpha} \hat{\rho}_{\nu}^{n+1}(k), \forall n \geq 0,
$$

and moreover, we have $\hat{\rho}^{n+1}=\hat{\rho}_{\nu}^{n+1}$ for all $n \geq 0$. But, for general initial data, since $\hat{\rho}_{\nu}^{0}$ is different from $\hat{\rho}^{0}$, the asymptotic scheme is not the correct one because of this initial mismatch. Then when the initial data is not at equilibrium, the previous scheme is not $\mathrm{AP}$ and a suitable modification has to be done to recover the AP property. To do so, we modify (3.35) to ensure that the first step of the asymptotic scheme writes (when $\varepsilon \rightarrow 0$ )

$$
\frac{\hat{\rho}_{\nu}^{1}(k)-\hat{\rho}^{0}(k)}{\Delta t}=-\bar{\kappa}|k|^{\alpha} \hat{\rho}_{\nu}^{1}
$$

Hence, we have to find an expression for the term $\hat{\rho}_{\nu}^{0}$ appearing in the first equation of (3.35) for $n=0$. Starting from (3.32) for $n=0$, we get, up to first order

$$
\hat{\rho}^{1}(k)=\left\langle\mathrm{e}^{-\frac{\Delta t}{\varepsilon^{\alpha}}(\nu+\mathrm{i} \varepsilon k \cdot v)} \hat{f}^{0}\right\rangle_{N_{v}, V}+d \hat{\rho}_{\nu}^{0}(k),
$$

where $d$ is given by

$$
d=\left\langle\frac{1-\mathrm{e}^{-\frac{\Delta t}{\varepsilon^{\alpha}}(\nu+\mathrm{i} \varepsilon k \cdot v)}}{\nu+\mathrm{i} \varepsilon k \cdot v} \nu M\right\rangle_{N_{v}, V} .
$$

Up to an error of order $\Delta t$, it is possible to replace $\hat{\rho}^{1}$ by $\hat{\rho}^{0}$ so that $\hat{\rho}_{\nu}^{0}$ becomes

$$
\hat{\rho}_{\nu}^{0}=\frac{1}{d}\left(\hat{\rho}^{0}-\left\langle\mathrm{e}^{-\frac{\Delta t}{\varepsilon^{\alpha}}(\nu+\mathrm{i} \varepsilon k \cdot v)} \hat{f}^{0}\right\rangle_{N_{v}, V}\right) .
$$

This expression is injected in (3.35) for $n=0$ to compute $\hat{\rho}_{\nu}^{1}$. Then, for any $\varepsilon,(3.51)$ is a consistent approximation and when $\varepsilon$ goes to zero, $\hat{\rho}_{\nu}^{0}$ tends to $\hat{\rho}^{0}$ which ensures the AP property for any initial data.

Another defect in the previous scheme (3.35) is that, for a general initial data, it does not preserve the total mass. To overcome this, we add the following consistent step for $\hat{f}^{n+1}$

$$
\hat{f}^{n+1}(k=0) \leftarrow \hat{f}^{n+1}(k=0)-\left\langle\hat{f}^{n+1}(k=0)\right\rangle_{N_{v}, V} M+\left\langle\hat{f}^{0}(k=0)\right\rangle_{N_{v}, V} M .
$$

Eventually, for a general initial condition, we have the following proposition 
Proposition 3.4. We consider the scheme defined for all $k$ and all time indices $1 \leq n \leq N, n \Delta t=T$ by (3.35)-(3.52) and for the first step by

$$
\left\{\begin{array}{l}
\hat{\rho}_{\nu}^{1}(k)=\frac{1}{K-c_{0}}\left[\hat{A}_{0}\left(t_{1}, k\right)+\frac{b_{0}}{d}\left(\hat{\rho}^{0}-\left\langle\mathrm{e}^{-\frac{\Delta t}{\varepsilon^{\alpha}}(\nu+\mathrm{i} \varepsilon k \cdot v)} \hat{f}^{0}\right\rangle_{N_{v}, V}\right)\right] \\
\hat{f}^{1}(k, v)=\mathrm{e}^{-\frac{\Delta t}{\varepsilon^{\alpha}}(\nu+\mathrm{i} \varepsilon k \cdot v)} \hat{f}^{0}(k, v)+\nu M\left(\gamma \hat{\rho}_{\nu}^{1}(k)+\beta \hat{\rho}_{\nu}^{0}(k)\right) \\
\hat{f}^{1}(0, v):=\hat{f}^{1}(0, v)-\left\langle\hat{f}^{1}(0, v)\right\rangle_{N_{v}, V} M+\left\langle\hat{f}^{0}(0, v)\right\rangle_{N_{v}, V} M,
\end{array}\right.
$$

with $K, \hat{A}_{0}, b_{0}, c_{0}, \gamma, \beta$ and $d$ defined in (3.21)-(3.30)-(3.31)-(3.34) and

(3.50). This scheme has the following properties:

(i) The scheme is first order in time and preserves the total mass.

(ii) The scheme is AP: for a fixed $\Delta t$, the scheme solves the anomalous diffusion equation when $\varepsilon$ goes to 0

$$
\frac{\hat{\rho}^{n+1}(k)-\hat{\rho}^{n}(k)}{\Delta t}=-\bar{\kappa}|k|^{\alpha} \hat{\rho}^{n+1}(k),
$$

where $\bar{\kappa}$ is defined in (3.6).

REMARK 6. for a general initial data, the time derivatives at $t=0$ are not bounded uniformly in $\varepsilon$. Hence, the uniform accuracy of the scheme is not a relevant property to be considered. However, the numerical tests tends to suggest that the consistency error is of order $\Delta t$ uniformly in $\varepsilon$.

Proof. To prove that the scheme is first order in time, we have to show that when $\varepsilon$ is fixed

$$
\left\|\hat{\rho}_{\nu}\left(t_{1}, \cdot\right)-\hat{\rho}_{\nu}^{1}(\cdot)\right\|_{\infty} \leq C_{\varepsilon} \Delta t
$$

where $C_{\varepsilon}$ is a constant when $\varepsilon$ is fixed. We will then be able to use the proof of Prop. 3.3. At the continuous level, (3.32) gives

$$
\hat{\rho}\left(t_{1}, k\right)=\left\langle\mathrm{e}^{-\frac{\Delta t}{\varepsilon^{\alpha}}(\nu+\mathrm{i} \varepsilon k \cdot v)} \hat{f}_{0}(k, v)\right\rangle_{V}+\int_{0}^{\frac{\Delta t}{\varepsilon^{\alpha}}}\left\langle\nu M \mathrm{e}^{-s(\nu+\mathrm{i} \varepsilon k \cdot v)}\right\rangle_{V} \hat{\rho}_{\nu}\left(t_{1}-\varepsilon^{\alpha} s, k\right) \mathrm{d} s .
$$

Denoting by $G(s)=\int_{0}^{t_{1}-\varepsilon^{\alpha} s} \partial_{t} \hat{\rho}_{\nu}(u) \mathrm{d} u$ and $H=-\int_{0}^{\Delta t} \partial_{t} \hat{\rho}(u) \mathrm{d} u$ the remainders of the Taylor expansions of $\hat{\rho}_{\nu}\left(t_{1}-\varepsilon^{\alpha} s, k\right)$ and $\hat{\rho}\left(t_{1}, k\right)$, we have

$$
\begin{aligned}
\hat{\rho}\left(t_{0}, k\right) & =\left\langle\mathrm{e}^{-\frac{\Delta t}{\varepsilon \alpha}(\nu+\mathrm{i} \varepsilon k \cdot v)} \hat{f}_{0}(k, v)\right\rangle_{V}+d \hat{\rho}_{\nu}\left(t_{0}, k\right)+H \\
& +\int_{0}^{\frac{\Delta t}{\varepsilon \alpha}}\left\langle\nu M \mathrm{e}^{-s(\nu+\mathrm{i} \varepsilon k \cdot v)}\right\rangle_{V} G(s) \mathrm{d} s,
\end{aligned}
$$

with $d$ defined in (3.50). Hence, the error made by replacing $\hat{\rho}_{\nu}\left(t_{0}, k\right)$ by its approximation (3.51) in the first iteration of (3.35) is of magnitude

$$
\frac{1}{d}\left(H+\int_{0}^{\frac{\Delta t}{\varepsilon x}}\left\langle\nu M \mathrm{e}^{-s(\nu+\mathrm{i} \varepsilon k \cdot v)}\right\rangle_{V} G(s) \mathrm{d} s\right) .
$$


Eventually, the error done in the first iteration of (3.53) writes

$$
\begin{aligned}
\left\|\hat{\rho}_{\nu}\left(t_{1}, \cdot\right)-\hat{\rho}_{\nu}^{1}(\cdot)\right\|_{\infty} & \leq\left|\frac{1}{d} \frac{b_{0}}{K-c_{0}}\left(H+\int_{0}^{\frac{\Delta t}{\varepsilon \alpha}}\left\langle\nu M \mathrm{e}^{-s(\nu+\mathrm{i} \varepsilon k \cdot v)}\right\rangle_{V} G(s) \mathrm{d} s\right)\right| \\
& +\left|\frac{F}{K-c_{0}}\right| \Delta t^{2},
\end{aligned}
$$

with $K, b_{0}, c_{0}$ defined in (3.21)-(3.25)-(3.26) and $F$ in (3.37) for $n=0$. The proof of Prop. 3.3 gives estimates for $1 /\left(K-c_{0}\right)$ and $F /\left(K-c_{0}\right)$ in (3.41) and (3.45). Moreover, since

$$
\left|b_{0}\right| \leq\left|\int_{0}^{\frac{\Delta t}{\varepsilon \alpha}}\left\langle\nu^{2} M \mathrm{e}^{-s(\nu+\mathrm{i} \varepsilon k \cdot v)}\right\rangle_{V} \mathrm{~d} s\right|,
$$

an exact computation of this integral shows that when $\varepsilon$ is fixed, the term $b_{0} / d$ is bounded. Assuming that the time derivatives of $\hat{\rho}$ and $\hat{\rho}_{\nu}$ are bounded, there exists a constant $C_{\varepsilon}$ depending a priori of $\varepsilon$ such that $\left\|\hat{\rho}_{\nu}\left(t_{1}, \cdot\right)-\hat{\rho}_{\nu}^{1}(\cdot)\right\|_{\infty} \leq C_{\varepsilon} \Delta t$. The proof of Prop. 3.3 can then be used to show that the scheme is of order 1 .

To show that (3.53) enjoys the AP property, we freeze $\Delta t$ and let $\varepsilon$ go to 0 in the expression of the scheme. The first iteration degenerates into

$$
\frac{\hat{\rho}_{\nu}^{1}(k)-\hat{\rho}^{0}(k)}{\Delta t}=-\bar{\kappa}|k|^{\alpha} \hat{\rho}_{\nu}^{1}(k), \quad \hat{\rho}^{1}(k, v)=\hat{\rho}_{\nu}^{1}(k) M,
$$

then, when $\varepsilon$ goes to zero $\hat{\rho}^{1}(k)=\hat{\rho}_{\nu}^{1}(k)$ which ensures that the first iteration of the scheme enjoys the AP property. As the following iterations are the same as in Prop. 3.3 , this proves (ii).

4. Numerical results. In this section, we present some numerical tests to validate the three schemes we proposed in Section 3. For simplicity, we denote by IS the the implicit scheme, MMS the micro-macro scheme and DS the scheme based on the Duhamel formulation of the kinetic equation. Eventually, the implicit Euler scheme for the anomalous diffusion equation (3.8) is denoted adiff. We use the equilibrium (1.2), the collision frequency (1.3) and the initial data

$$
f_{0}(x, v)=(1+\sin (\pi x)) M_{0},
$$

for $x \in[-1,1]$ and $v \in V=[-1,1]$ and $w_{\max }=1$ for the computation of $\bar{\kappa}$ in (3.6). The normalization constant $M_{0}$ is chosen such that $\langle M\rangle_{N_{v}, V}=1$ and $\nu_{0}$ is fixed $\nu_{0}=1$. We consider periodic conditions for the space, then the discretization used for the space variable uses $N_{x}+1$ points and is given by

$$
x_{i}=-1+i \Delta x, \quad 0 \leq i \leq N_{x},
$$

where $\Delta x=\frac{2}{N_{x}}$. This space discretization is linked to the Fourier modes we use for the discrete Fourier transform in the numerical computations, that are the integers $k$ such that $-N_{x} / 2 \leq k \leq N_{x} / 2$. The uniform discretization of the velocities is done with $N_{v}=2 N_{v}^{\prime}+1$ points symmetrically distributed to ensure that the property $\langle v M\rangle_{N_{v}, V}=0$ is conserved at the discrete level. Denoting $\Delta v=1 / N_{v}^{\prime}$, we set

$$
v_{j}=-1+j \Delta v, \quad 0 \leq j \leq 2 N_{v}^{\prime} .
$$

Since the schemes do not require a refined velocity grid to enjoy the AP property, we fix $N_{v}^{\prime}=10$ in all the following tests. All the tests are performed using a final time $T=1$ and $\beta=1$, the time step $\Delta t$ being specified in each case. 
4.1. The implicit scheme (IS). In this part, we test the properties of the IS scheme. To highlight its AP character, we present in the left hand side of Fig. 4.1 the densities $\rho(T, x)$ obtained by IS for $\Delta t=10^{-1}, N_{x}=32$ and a range of $\varepsilon$. For this figure, we compare our results to the anomalous diffusion limit given by the adiff scheme with the same time step. It appears that the densities converge slowly to the anomalous diffusion limit when $\varepsilon$ goes to 0 . In the right part of Fig. 4.1, we plot the $L^{\infty}$ norm (in space) of the error between $\rho(T, x)$ given by IS and $\rho_{\text {adiff }}(T, x)$ given by adiff as a function of $\varepsilon$ (in $\log$ scale). Then, we can compare our numerical results to the rate of convergence computed in Prop. 2.2. We observe that the numerical rate 0.334 is in a very good agreement with the theoretical rate $d /(d+1+\beta) \approx 0.333$. Let us remark that we showed in Prop. 2.2 that theoretically, the convergence happens rather with rate $\varepsilon^{\min \left(\frac{d}{d+1+\beta}, \frac{\beta}{d+1+\beta}\right)}$, but the terms (5.1)-(5.2) giving the $O\left(\varepsilon^{\frac{\beta}{d+1+\beta}}\right)$ do not arise numerically. Indeed, it is given by a divergent integral multiplied by a power of $\varepsilon$. As we use a bounded domain for $v$ and we did not apply a specific treatment for these terms, this divergent integral is numerically finite and then it vanishes when $\varepsilon$ becomes small.
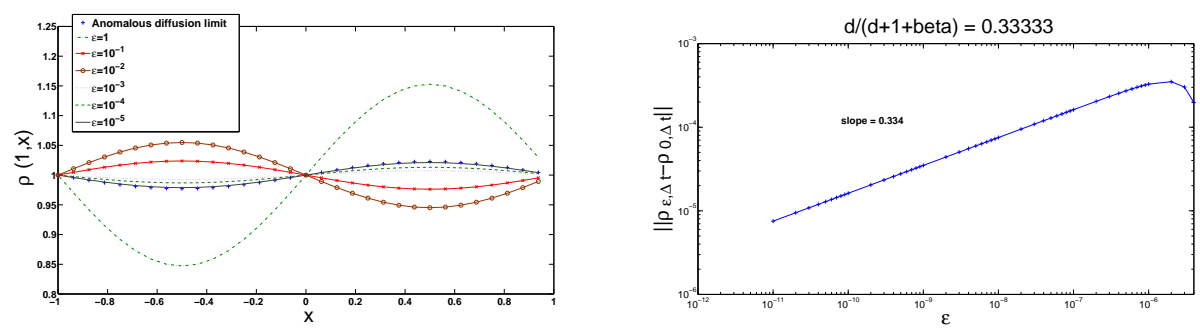

FIG. 4.1. Left: For $\Delta t=10^{-1}$, the densities given by IS for a range of $\varepsilon$ and by adiff. Right: For $\Delta t=10^{-1}$, the error between the densities given by IS and by adiff as a function of $\varepsilon$ (log scale).

4.2. Micro-macro based scheme (MMS). In this part, we test the properties of MMS. In the left hand side of Fig. 4.2 we present the densities $\rho(T, x)$ obtained with the MMS scheme for a range of $\varepsilon$ and we compare it to the anomalous diffusion limit given by adiff. We used $N_{x}=32$ points to discretize the space. We use Fourier variable for the equation on $\rho$ (macro part), but the space derivatives of the micro part are discretized with a classical first order upwind scheme. Hence, a CFL condition has to respected so that $\Delta t=10^{-3}$. When $\varepsilon$ goes to zero, the densities obtained by MMS converges towards the density obtained by adiff. On the right hand side of Fig. 4.2, we plot the $L^{\infty}$ norm (in space) of the error between $\rho(T, x)$ obtained by MMS and $\rho_{\text {adiff }}(T, x)$ obtained by adiff (using $\Delta t=10^{-3}$ ), as a function of $\varepsilon$. We can observe that the convergence happens with a rate 0.337 which is very close to the theoretical value $\varepsilon^{\frac{d}{d+1+\beta}}=0.333$. 

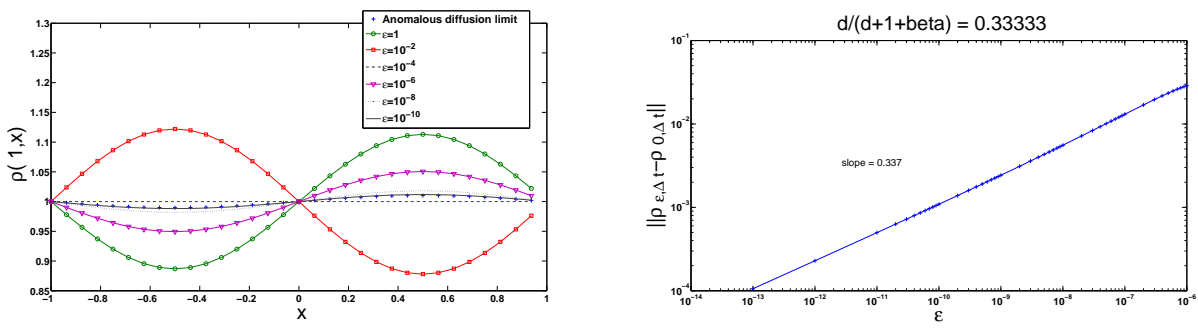

FIG. 4.2. left: For $\Delta t=10^{-3}$, the densities given by the MMS scheme for a range of $\varepsilon$ and the anomalous diffusion limit given by the adiff scheme. Right: For $\Delta t=10^{-3}$, the error between the $M M S$ scheme and the DSA scheme as a function of $\varepsilon$ (log scale).

4.3. The integral formulation scheme (DS). In this section, we test the properties of the DS scheme. First of all, we remark that for $\varepsilon=1$ the scheme appears to be of second order, as suggested by Fig. 4.3. For this test, we consider $N_{x}=8$ and we consider the $L^{\infty}$ norm (in space) of the error between the densities given by DS for a range of $\Delta t \in\left[2 \times 10^{-3}, 0.5\right]$ and the density given by DS for $\Delta t=10^{-3}$ (which is the reference).

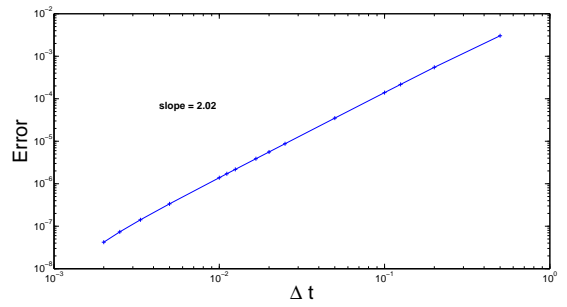

FIG. 4.3. For $\varepsilon=1$, the error between DS computed for $\Delta t=10^{-3}$ and $D S$ computed for a range of $\Delta t \in\left[2 \times 10^{-3}, 0.5\right]$ (log scale).

Then, we study the AP character of the scheme, fixing $\Delta t=10^{-1}$, considering a range of $\varepsilon$. As previously, on the left hand side of Fig. 4.4, the densities $\rho(T, x)$ obtained by DS and by adiff (using $\Delta t=10^{-1}$ ) are plotted as a function of space with $N_{x}=32$. On the right hand side of Fig. 4.4, the $L^{\infty}$ norm (in space) of the error between $\rho(T, x)$ obtained by DS and $\rho_{\text {adiff }}(T, x)$ obtained by adiff (using $\Delta t=10^{-1}$ ) is plotted as a function of $\varepsilon$, for $N_{x}=8$. As in the previous cases, we observe that the numerical convergence rate is close to the theoretical one $\varepsilon^{\frac{d}{d+1+\beta}}$.

Eventually, we illustrate that DS is first order in time uniformly in $\varepsilon$. Indeed, the left hand side of Fig. 4.5 displays the $L^{\infty}$ error (in space)

$$
E(\varepsilon, \Delta t)=\left\|\rho_{\Delta t}^{\varepsilon}(T, \cdot)-\rho_{\Delta t=10^{-3}}^{\varepsilon}(T, \cdot)\right\|_{\infty},
$$

where $\rho_{\Delta t=10^{-3}}^{\varepsilon}(T, x)$ is obtained by DS with $\Delta t=10^{-3}$ and $\rho_{\Delta t}^{\varepsilon}(T, \cdot)$ is obtained by DS using larger $\Delta t$. The error $E(\varepsilon, \Delta t)$ is computed for a range of $\Delta t \in\left[5 \times 10^{-3}, 0.5\right]$ and of $\varepsilon \in\left[10^{-7}, 1\right]$. For this test, we fixed $N_{x}=8$. These errors are stratified with respect to $\Delta t$, confirming the uniform accuracy of DS with respect to $\varepsilon$.

We now consider the following non equilibrium initial data

$$
f_{0}(x, v)=(1+\sin (\pi x)) \mathrm{e}^{-|v|},
$$



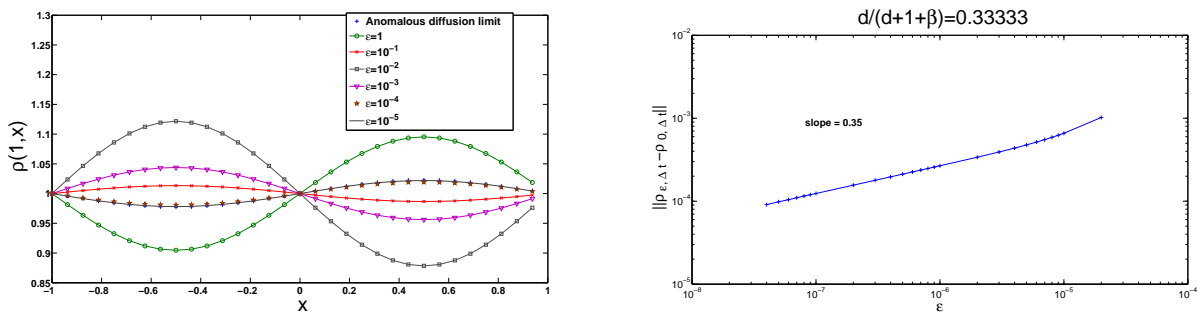

Fig. 4.4. Left: For $\Delta t=10^{-1}$, the densities given by $D S$ for a range of $\varepsilon$ and by adiff. Right: For $\Delta t=10^{-1}$, the error between $D S$ and the adiff as a function of $\varepsilon$ (log scale).

and the same numerical parameters as in the last test. As previously, we also look at (4.4) (see the right hand side of Fig. 4.5). It turns out that even if the initial condition is out of equilibrium, the Duhamel based scheme is uniformly accurate. As a consequence, considering the maximum with respect to $\varepsilon \in\left[10^{-7}, 1\right]$ of $E(\varepsilon, \Delta t)$ enables to highlight that DS is of order 1 uniformly in $\varepsilon$ (obviously, the same occurs for the well-prepared initial data).
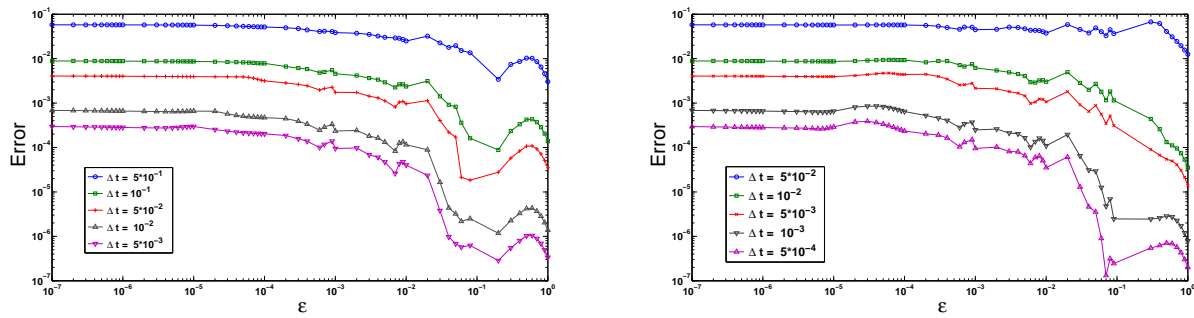

FIG. 4.5. The error between $D S$ computed for $\Delta t=10^{-3}$ and $D S$ computed for a range of $\Delta t$, as functions of $\varepsilon$ (log scale). Left: equilibrium initial data (4.1). Right: non equilibrium initial data (4.5).

5. Appendix. In this section, we deal with the proof of Prop. 2.1.

The proof is a direct consequence of the following lemma:

Lemma 5.1. Let $\hat{f}$ be the solution of (1.5) in the Fourier variable for a given initial condition $\hat{f}_{0}$ and let $t>0$ be fixed. Then its modified density $\hat{\rho}_{\nu}$ satisfies formally

$$
\partial_{t} \hat{\rho}_{\nu}(t, k)+|k|^{\alpha} \kappa \hat{\rho}_{\nu}(t, k)=O\left(\varepsilon^{\frac{d}{d+1+\beta}}\right)+O\left(\varepsilon^{\frac{\beta}{d+1+\beta}}\right),
$$

with $\kappa$ given by $(1.7)$ and with the initial condition $\hat{\rho}_{\nu}(0, k)=\left\langle\nu \hat{f}_{0}(k, v)\right\rangle_{V} /\langle\nu M\rangle_{V}$. Furthermore, the density and modified density $\hat{\rho}$ and $\hat{\rho}_{\nu}$ satisfy formally $\hat{\rho}(t, k)=$ $\hat{\rho}_{\nu}(t, k)+O\left(\varepsilon^{\alpha-\gamma}\right)$, for all $0<\gamma<\alpha$.

Proof. (Lemma 5.1) The proof relies on the integral formulation (3.19) which gives the expression (3.20) after an integration in $v \in V$. Using the notations $K$ and 
$\hat{A}_{0}$ defined in (3.21), let us rewrite (3.20) at time $t$ as follows

$$
\begin{aligned}
& K \hat{\rho}_{\nu}(t, k)=\hat{A}_{0}(t, k)+\int_{0}^{\frac{t}{\varepsilon^{\alpha}}}\left\langle\nu^{2} M \mathrm{e}^{-\nu s}\right\rangle_{V}\left(\hat{\rho}_{\nu}\left(t-\varepsilon^{\alpha} s, k\right)-\hat{\rho}_{\nu}(t, k)\right) \mathrm{d} s \\
& +\int_{0}^{\frac{t}{\varepsilon^{\alpha}}}\left\langle\nu^{2} M\left(e^{-s(\nu+\mathrm{i} \varepsilon k \cdot v)}-\mathrm{e}^{-\nu s}\right)\right\rangle_{V}\left(\hat{\rho}_{\nu}\left(t-\varepsilon^{\alpha} s, k\right)-\hat{\rho}_{\nu}(t, k)\right) \mathrm{d} s \\
& +\int_{0}^{\frac{t}{\varepsilon^{\alpha}}}\left\langle\nu^{2} M\left(e^{-s(\nu+\mathrm{i} \varepsilon k \cdot v)}-\mathrm{e}^{-\nu s}\right)\right\rangle_{V} \mathrm{~d} s \hat{\rho}_{\nu}(t, k) \\
& +\int_{0}^{\frac{t}{\varepsilon^{\alpha}}}\left\langle\nu^{2} M \mathrm{e}^{-\nu s}\right\rangle_{V} \mathrm{~d} s \hat{\rho}_{\nu}(t, k),
\end{aligned}
$$

and consider each line separately. First of all, let us remark that in (5.4), the integral in $s$ can be directly computed and that the initial layer term $\hat{A}_{0}$ in (5.1) is exponentially small. Let us denote $\mathcal{I}_{1}$ the integral term appearing in (5.1) and show that is equivalent to $-\varepsilon^{\alpha} \partial_{t} \hat{\rho}_{\nu}(t, k)$ for small $\varepsilon$ up to a rest of order $O\left(\varepsilon^{\alpha+\frac{\alpha d}{d+2+\beta}}\right)$. We rewrite $\mathcal{I}_{1}$ as

$$
\mathcal{I}_{1}=-\int_{0}^{\frac{t}{\varepsilon^{\alpha}}}\left\langle\nu^{2} M \mathrm{e}^{-\nu s}\right\rangle_{V}\left(\int_{t-\varepsilon^{\alpha} s}^{t} \partial_{t} \hat{\rho}_{\nu}(\xi, k) \mathrm{d} \xi\right) \mathrm{d} s
$$

and we permute the integrals before computing exactly the integral in $s$ to get

$$
\mathcal{I}_{1}=-\int_{0}^{t} \partial_{t} \hat{\rho}_{\nu}(t-\xi, k)\left\langle\nu M \mathrm{e}^{-\nu \frac{\xi}{\varepsilon^{\alpha}}}\right\rangle_{V} \mathrm{~d} \xi+O\left(\varepsilon^{\infty}\right) .
$$

Hence, up to an exponentially small error in $\varepsilon, \mathcal{I}_{1}$ reads

$$
\mathcal{I}_{1}=-\varepsilon^{\alpha} \partial_{t} \hat{\rho}_{\nu}(t, k)-\varepsilon^{\alpha} \int_{0}^{\frac{t}{\varepsilon^{\alpha}}}\left\langle\nu M \mathrm{e}^{-\nu(v) \xi}\right\rangle_{V}\left(\partial_{t} \hat{\rho}_{\nu}\left(t-\varepsilon^{\alpha} \xi, k\right)-\partial_{t} \hat{\rho}_{\nu}(t, k)\right) \mathrm{d} \xi .
$$

It remains to show that the second term appearing in $\mathcal{I}_{1}$ is smaller that $\varepsilon^{\alpha}$. Indeed in the brackets we do the change of variables $w=\left(\nu_{0} \xi\right)^{\frac{1}{d+2+\beta}} v$ to make appear a fractional time derivative. Then with the change of variables $\xi \rightarrow \varepsilon^{\alpha} \xi$ in the integral in $\xi, \mathcal{I}_{1}$ writes

$$
\begin{aligned}
& \mathcal{I}_{1}=-\varepsilon^{\alpha} \partial_{t} \hat{\rho}_{\nu}(t, k)+O\left(\varepsilon^{\infty}\right) \\
& -\varepsilon^{\alpha\left(\frac{2 d+2+\beta}{d+2+\beta}\right)} M_{0} \nu_{0}^{-\frac{d}{d+2+\beta}} \int_{0}^{t}\left\langle|w|^{d+2+\beta} \mathrm{e}^{-|w|^{d+2+\beta}}\right\rangle_{|w| \leq \tilde{\delta}} \frac{\partial_{t} \hat{\rho}_{\nu}(t-\xi, k)-\partial_{t} \hat{\rho}_{\nu}(t, k)}{\xi^{\frac{2 d+2+\beta}{d+2+\beta}}} \mathrm{d} \xi
\end{aligned}
$$

where $\tilde{\delta}=\left(\frac{\nu_{0} \xi}{\varepsilon^{\alpha}}\right)^{\frac{1}{d+2+\beta}}$ and $\langle\cdot\rangle_{|w| \leq \tilde{\delta}}$ denotes the integration in $w$ on the domain $|w| \leq$ $\tilde{\delta}$. The term into brackets is integrable on $\mathbb{R}^{d}$ and $\frac{2 d+2+\beta}{d+2+\beta}=1+\frac{d}{d+2+\beta} \in(1,2)$. Supposing that $\hat{\rho}_{\nu}$ is regular, the integral in $\xi$ is well defined and is bounded by a constant for any fixed $t$. Hence

$$
\mathcal{I}_{1}=-\varepsilon^{\alpha} \partial_{t} \hat{\rho}_{\nu}(t, k)+O\left(\varepsilon^{\alpha+\frac{\alpha d}{d+2+\beta}}\right) .
$$

Now, let us denote $\mathcal{I}_{2}$ the term (5.2) and remark that it vanishes for $k=0$. We want to show that it is of magnitude $O\left(\varepsilon^{\alpha+\frac{d}{d+1+\beta}}\right)$ when $\varepsilon$ goes to 0 . For nonzero 
$k$ we apply the change of variable $w=\frac{\varepsilon|k| v}{\nu(v)}$, such that $v=\left(\frac{\varepsilon|k|}{\nu_{0}|w|^{d+2+\beta}}\right)^{\frac{1}{d+1+\beta}} w$ and $\mathrm{d} v=\frac{1}{d+1+\beta}\left(\frac{\varepsilon|k|}{\nu_{0}|w|^{d+2+\beta}}\right)^{\frac{d}{d+1+\beta}} \mathrm{d} w$, to make the limit appear in the brackets. Then, with a Taylor expansion of $\hat{\rho}_{\nu}, \mathcal{I}_{2}$ writes

$$
\mathcal{I}_{2}=-\varepsilon^{\alpha} C \int_{0}^{\frac{t}{\varepsilon^{\alpha}}}\left\langle\frac{\varphi}{|w|^{d+\alpha}}\left(\mathrm{e}^{-s \varphi(1+\mathrm{i} w \cdot e)}-\mathrm{e}^{-s \varphi}\right)\right\rangle_{|w| \geq \Delta}\left(\int_{t-\varepsilon^{\alpha} s}^{t} \partial_{t} \hat{\rho}_{\nu}(\xi, k) \mathrm{d} \xi\right) \mathrm{d} s
$$

where $C=\frac{|k|^{\alpha} M_{0} \nu_{0}^{1-\alpha}}{d+1+\beta}, \varphi=\nu_{0}\left(\frac{\varepsilon|k|}{\nu_{0}|w|}\right)^{\frac{d+2+\beta}{d+1+\beta}}, \Delta=\frac{\varepsilon|k|}{\nu_{0}}$ and where $e$ denotes any unitary vector. As for $\mathcal{I}_{1}$, we permute the integrals before computing the integral in $s$ to get the following expression of $\mathcal{I}_{2}$

$$
\mathcal{I}_{2}=\varepsilon^{2 \alpha} C \mathcal{I}_{2}^{1}+\varepsilon^{\alpha} C \partial_{t} \hat{\rho}_{\nu}(t, k) \mathcal{I}_{2}^{2}+O\left(\varepsilon^{\infty}\right),
$$

with

$$
\begin{aligned}
& \mathcal{I}_{2}^{1}=\int_{0}^{\frac{t}{\varepsilon^{\alpha}}}\left\langle\frac{1}{\mid w^{d+\alpha}}\left(\frac{\mathrm{e}^{-\xi \varphi(1+\mathrm{i} w \cdot e)}}{1+\mathrm{i} w \cdot e}-\mathrm{e}^{-\xi \varphi}\right)\right\rangle_{|w| \geq \Delta}\left(\partial_{t} \hat{\rho}_{\nu}\left(t-\varepsilon^{\alpha} \xi, k\right)-\partial_{t} \hat{\rho}_{\nu}(t, k)\right) \mathrm{d} \xi \\
& \mathcal{I}_{2}^{2}=\int_{0}^{t}\left\langle\frac{1}{|w|^{d+\alpha}}\left(\frac{\mathrm{e}^{-\frac{\xi}{\varepsilon^{\alpha}} \varphi(1+\mathrm{i} w \cdot e)}}{1+\mathrm{i} w \cdot e}-\mathrm{e}^{-\frac{\xi}{\varepsilon^{\alpha}} \varphi}\right)\right\rangle_{|w| \geq \Delta} \mathrm{d} \xi .
\end{aligned}
$$

The exact computation of the integral in $\xi$ leads to

$$
\mathcal{I}_{2}^{2}=O\left(\varepsilon^{\frac{d}{d+1+\beta}}\right)
$$

and we now focus on $\mathcal{I}_{2}^{1}$. In the brackets we do the change of variables $v=\frac{\nu_{0}}{|k| \varepsilon}\left(\xi \nu_{0}\right)^{-\frac{d+1+\beta}{d+2+\beta}}$ such that $\xi \varphi=\frac{1}{|v|^{\frac{d+2+\beta}{d+1+\beta}}}$, to get

$$
\begin{aligned}
& \left\langle\frac{1}{|w|^{d+\alpha}}\left(\frac{\mathrm{e}^{-\frac{\xi}{\varepsilon^{\alpha}} \varphi(1+\mathrm{i} w \cdot e)}}{1+\mathrm{i} w \cdot e}-\mathrm{e}^{-\frac{\xi}{\varepsilon^{\alpha}} \varphi}\right)\right\rangle_{|w| \geq \Delta} \\
& =\frac{\nu_{0}^{\frac{\alpha}{d+2+\beta}}}{\varepsilon^{\alpha}|k|^{\alpha}} \frac{1}{\xi^{\alpha \frac{d+1+\beta}{d+2+\beta}}}\left\langle\frac{1}{|v|^{d+\alpha}}\left(\frac{\mathrm{e}^{-\frac{1}{|v|^{\frac{d+2+\beta}{d+1+\beta}}}(1+\mathrm{i} \psi(v) \cdot e)}}{1+\mathrm{i} \psi(v) \cdot e}-\mathrm{e}^{-\frac{1}{|v|^{\frac{d+2+\beta}{d+1+\beta}}}}\right)\right\rangle_{|v| \geq \tilde{\Delta}},
\end{aligned}
$$

with $\psi(v)=\frac{\varepsilon|k|}{\nu_{0}}\left(\xi \nu_{0}\right)^{\frac{d+1+\beta}{d+2+\beta}} v$ and $\tilde{\Delta}=\frac{\nu_{0}}{\varepsilon|k|}\left(\xi \nu_{0}\right)^{-\frac{d+1+\beta}{d+2+\beta}} \Delta$. This last expression gives the following upper bound for $\mathcal{I}_{2}^{1}$

$$
\left|\varepsilon^{\alpha} \mathcal{I}_{2}^{1}\right| \leq C \int_{0}^{\frac{t}{\varepsilon^{\alpha}}} \frac{\left|\partial_{t} \hat{\rho}_{\nu}\left(t-\varepsilon^{\alpha} \xi, k\right)-\partial_{t} \hat{\rho}_{\nu}(t, k)\right|}{|\xi|^{\alpha \frac{d+1+\beta}{d+2+\beta}}} \mathrm{d} \xi
$$

we then apply the change of variables $\xi \rightarrow \varepsilon^{\alpha} \xi$ to get $\varepsilon^{\alpha} \mathcal{I}_{2}^{1}=O\left(\varepsilon^{\frac{\alpha d}{d+2+\beta}}\right)$, which combined with (5.9) in (5.6) leads to

$$
\mathcal{I}_{2}=O\left(\varepsilon^{\alpha+\frac{d}{d+1+\beta}}\right)+O\left(\varepsilon^{\alpha+\frac{\alpha d}{d+2+\beta}}\right)=O\left(\varepsilon^{\alpha+\frac{d}{d+1+\beta}}\right) .
$$


It remains to consider (5.3), let us denote

$$
\mathcal{I}_{3}=\int_{0}^{\frac{t}{\varepsilon \alpha}}\left\langle\nu^{2} M\left(\mathrm{e}^{-s(\nu+\mathrm{i} \varepsilon k \cdot v)}-\mathrm{e}^{-\nu s}\right)\right\rangle_{V} \mathrm{~d} s
$$

that writes after the change of variables $w=\frac{\varepsilon|k| v}{\nu(v)}$ and an exact integration in $s$

$$
\mathcal{I}_{3}=-\frac{\varepsilon^{\alpha}|k|^{\alpha} \nu_{0}^{1-\alpha} M_{0}}{d+1+\beta}\left\langle\frac{1}{|w|^{d+\alpha}} \frac{(w \cdot e)^{2}}{1+(w \cdot e)^{2}}\right\rangle_{|w| \geq \Delta}+O\left(\varepsilon^{\infty}\right)
$$

where we see the anomalous diffusion coefficient appear. Eventually, we get the following equivalent for $\mathcal{I}_{3}$

$$
\mathcal{I}_{3}=-\varepsilon^{\alpha} \kappa+O\left(\varepsilon^{\alpha+\frac{\beta}{d+1+\beta}}\right),
$$

with $\kappa$ given by (1.7). Finally replacing the lines (5.1)-(5.2)-(5.3)-(5.4) with their equivalents leads to the result

$$
\partial_{t} \hat{\rho}_{\nu}+|k|^{\alpha} \kappa \hat{\rho}_{\nu}=O\left(\varepsilon^{\frac{d}{d+1+\beta}}\right)+O\left(\varepsilon^{\frac{\beta}{d+1+\beta}}\right) .
$$

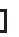

\section{REFERENCES}

[1] C. Bardos, R. Santos, and R. Sentis. Diffusion approximation and computation of the critical size. Trans. Amer. Math. Soc., 284(2):617-649, 1984.

[2] N. Ben Abdallah, A. Mellet, and M. Puel. Anomalous diffusion limit for kinetic equations with degenerate collision frequency. Math. Models Methods Appl. Sci., 21(11):2249-2262, 2011.

[3] N. Ben Abdallah, A. Mellet, and M. Puel. Fractional diffusion limit for collisional kinetic equations : A Hilbert expansion approach. Kinetic and related models, 4(4):873-900, 2011.

[4] A. Bensoussan, J. L. Lions, and G. Papanicolaou. Boundary layers and homogenization of transport processes. Publ. Res. Int. Math. Sci., 15:53-157, 1979.

[5] A.V. Bobylev, J.A. Carrillo, and I.M. Gamba. On some properties of kinetic and hydrodynamic equations for inelastic interactions. J. Stat. Phys., 98:743-773, 2000.

[6] A.V. Bobylev and I.M. Gamba. Boltzmann equations for mixtures of maxwell gases: Exact solutions and power like tails. J. Stat. Phys., 124(2-4):497-516, 2006.

[7] J.A. Carrillo, T. Goudon, P. Lafitte, and F. Vecil. Numerical schemes of diffusion asymptotics and moment closures for kinetic equations. J. Sci. Comput., 36(1):113-149, 2008.

[8] N. Crouseilles, H. Hivert, and M. Lemou. Multiscale numerical schemes for kinetic equations in the anomalous diffusion limit. Comptes Rendus Mathematique, 353(8):755 - 760, 2015.

[9] N. Crouseilles, H. Hivert, and M. Lemou. Numerical schemes for kinetic equations in the anomalous diffusion limit. Part I: the case of heavy-tailed equilibrium. arXiv:1503.04586, 2015.

[10] P. Degond, T. Goudon, and F. Poupaud. Diffusion limit for non homogeneous and non-microreversible processes. Indiana Univ. Math. J, 49:1175-1198, 2000.

[11] D. del Castillo-Negrete, B. Carreras, and V. Lynch. Non-diffusive transport in plasma turbulence: A fractional diffusion approach. Phys. Rev. Lett., 94:065003, Feb 2005.

[12] M.H. Ernst and R. Brito. Scaling solutions of inelastic Boltzmann equations with overpopulated high energy tails. J. Stat. Phys., 109(3-4):407-432, 2002.

[13] M. Jara, T. Komorowski, and S. Olla. Limit theorems for additive functionals of a Markov chain. Ann. Appl. Probab., 19(6):2270-2300, 122009.

[14] S. Jin. Efficient asymptotic-preserving (AP) schemes for some multiscale kinetic equations. SIAM J. Sci. Comput., 21(2):441-454, 1999.

[15] S Jin, L. Pareschi, and G. Toscani. Uniformly accurate diffusive relaxation schemes for multiscale transport equations. SIAM J. Numer. Anal., 38(3):913-936, 2000.

[16] A. Klar. An asymptotic-induced scheme for nonstationary transport equations in the diffusive limit. SIAM J. Numer. Anal., 35(3):1073-1094, 1998. 
[17] C. Kleiber and S. Kotz. Statistical size distributions in economics and actuarial sciences. John Wiley and Sons, 2003.

[18] E. W. Larsen and J. B. Keller. Asymptotic solution of neutron transport problems for small mean free paths. J. Math. Phys, 15:75-81, 1974.

[19] M. Lemou and F. Méhats. Micro-macro schemes for kinetic equations including boundary layers. SIAM J. Sci. Comput., 34(6):734-760, 2012.

[20] M. Lemou and L. Mieussens. A new asymptotic preserving scheme based on micro-macro formulation for linear kinetic equations in the diffusion limit. SIAM J. Sci. Comput., 31(1):334-368, 2008.

[21] A. Mellet. Fractional diffusion limit for collisional kinetic equations: A moments method. Indiana Univ. Math. J., 59:1333-1360, 2010.

[22] A. Mellet and S. Merino-Aceituno. Anomalous energy transport in FPU- $\beta$ chain. Journal of Statistical Physics, pages 1-39, 2015.

[23] A. Mellet, S. Mischler, and C. Mouhot. Fractional diffusion limit for collisional kinetic equations. Arch. Ration. Mech. Anal., 199:493-525, 2011.

[24] L. Mieussens. On the asymptotic preserving property of the unified gas kinetic scheme for the diffusion limit of linear kinetic models. J. Comput. Phys., 253:138-156, 2013.

[25] G. Naldi and L. Pareschi. Numerical schemes for kinetic equations in diffusive regimes. Appl. Math. Letters, 11(2):29 - 35, 1998.

[26] L. Wang and B. Yan. An asymptotic-preserving scheme for linear kinetic equation with fractional diffusion limit. Preprint, 2015.

[27] E. Wigner. Nuclear reactor theory. AMS, 1961. 